\title{
Evidence for Sub-Chandrasekhar Type Ia Supernovae from Stellar Abundances in Dwarf Galaxies*
}

\author{
Evan N. Kirby ${ }^{1}$ (10, Justin L. Xie ${ }^{2}$, Rachel Guo ${ }^{2}$, Mithi A. C. de los Reyes ${ }^{1}$, Maria Bergemann ${ }^{3}$ (10, Mikhail Kovalev ${ }^{3}$, \\ Ken J. Shen ${ }^{4}$ (D), Anthony L. Piro ${ }^{5}$ (i), and Andrew McWilliam ${ }^{5}$ (D) \\ ${ }^{1}$ California Institute of Technology, 1200 E. California Blvd., MC 249-17, Pasadena, CA 91125, USA; enk@astro.caltech.edu \\ ${ }^{2}$ Harvard College, 28 Fernald Dr., Cambridge, MA 02138, USA \\ ${ }^{3}$ Max-Planck Institute for Astronomy, D-69117, Heidelberg, Germany \\ ${ }^{4}$ Department of Astronomy and Theoretical Astrophysics Center, University of California, Berkeley, CA 94720, USA \\ ${ }^{5}$ The Observatories of the Carnegie Institution for Science, 813 Santa Barbara St., Pasadena, CA 91101, USA \\ Received 2019 April 26; revised 2019 June 18; accepted 2019 June 22; published 2019 August 12
}

\begin{abstract}
There is no consensus on the progenitors of Type Ia supernovae (SNe Ia) despite their importance for cosmology and chemical evolution. We address this question using our previously published catalogs of $\mathrm{Mg}, \mathrm{Si}, \mathrm{Ca}, \mathrm{Cr}, \mathrm{Fe}$, $\mathrm{Co}$, and $\mathrm{Ni}$ abundances in dwarf galaxy satellites of the Milky Way (MW) to constrain the mass at which the white dwarf (WD) explodes during a typical SN Ia. We fit a simple bi-linear model to the evolution of $[\mathrm{X} / \mathrm{Fe}]$ with $[\mathrm{Fe} / \mathrm{H}]$, where $\mathrm{X}$ represents each of the elements mentioned above. We use the evolution of $[\mathrm{Mg} / \mathrm{Fe}]$ coupled with theoretical supernova yields to isolate what fraction of the elements originated in SNe Ia. Then, we infer the $[\mathrm{X} / \mathrm{Fe}]$ yield of SNe Ia for all of the elements except Mg. We compare these observationally inferred yields to recent theoretical predictions for two classes of Chandrasekhar-mass $\left(M_{\mathrm{Ch}}\right) \mathrm{SN}$ Ia as well as sub- $M_{\mathrm{Ch}} \mathrm{SNe}$ Ia. Most of the inferred SN Ia yields are consistent with all of the theoretical models, but $[\mathrm{Ni} / \mathrm{Fe}]$ is consistent only with sub- $M_{\mathrm{Ch}}$ models. We conclude that the dominant type of SN Ia in ancient dwarf galaxies is the explosion of a sub- $M_{\mathrm{Ch}} \mathrm{WD}$. The MW and dwarf galaxies with extended star formation histories have higher $[\mathrm{Ni} / \mathrm{Fe}]$ abundances, which could indicate that the dominant class of SN Ia is different for galaxies where star formation lasted for at least several Gyr.
\end{abstract}

Key words: galaxies: abundances - galaxies: dwarf - Local Group - nuclear reactions, nucleosynthesis, abundances - supernovae: general

\section{Introduction}

Type Ia supernovae (SNe Ia) are some of the most important events in astrophysics. ${ }^{6}$ They are the basis for the Nobel Prizewinning measurement of cosmological acceleration (Riess et al. 1998; Perlmutter et al. 1999). They are also the origin of most of the iron and some of the elements adjacent in atomic number to iron (Fe-peak elements) in the Galaxy (Nomoto et al. 1984a). Despite their importance, there remain outstanding questions about the physics of the explosions and the nature of their progenitors. They result from the explosions of carbon/oxygen white dwarfs (WDs; Arnett 1969) in binary systems, but the details of the burning and even the numbers of WDs involved are hotly debated. Resolving these questions might provide a physical basis for the use of SNe Ia as standardizable candles (Phillips 1993), thus justifying their widespread use as cosmological tools.

Models of SNe Ia have been developed over the past five decades. The first models supposed that a WD grew in mass by the accretion of hydrogen from a red giant companion (Whelan \& Iben 1973). When the WD neared the Chandrasekhar mass $\left(M_{\mathrm{Ch}}\right)$, its core reached sufficiently high temperature and density to ignite carbon.

\footnotetext{
* The data presented herein were obtained at the W. M. Keck Observatory, which is operated as a scientific partnership among the California Institute of Technology, the University of California and the National Aeronautics and Space Administration. The Observatory was made possible by the generous financial support of the W. M. Keck Foundation.

6 See reviews by Yungelson \& Livio (2000), Hillebrandt et al. (2013), Maoz et al. (2014), Maguire (2017), and Seitenzahl \& Townsley (2017).
}

Nomoto et al. (1984b) first calculated the nucleosynthesis of $\mathrm{SNe}$ Ia exploding with a mass near $M_{\mathrm{Ch}}$. They found that the rate of carbon burning strongly affected the elements produced. If the WD detonated supersonically, i.e., on a timescale faster than the dynamical time, it produced Fe-group elements but almost no Si. In order to explain the Si present in the spectra of $\mathrm{SNe}$ Ia, Nomoto et al. invoked deflagration, whereby a carbonburning flame consumes the WD subsonically, i.e., more slowly than the dynamical time.

A hybrid of deflagration and detonation (Khokhlov 1991; Iwamoto et al. 1999) can achieve the appropriate balance of Si-group and Fe-group elements. In this model, an initial deflagration gives way to a detonation. The model is called "delayed detonation" or "deflagration-to-detonation transition" (DDT). The deflagration burns material at high density, but it also allows the WD to expand and become less dense before the detonation consumes the remainder of the carbon. The details of the nucleosynthesis depend heavily on the structure of the progenitor WD and the physics of the carbon ignition (e.g., Byrohl et al. 2019; Leung \& Nomoto 2018), as well as the implementation of the hydrodynamics and the dimensionality of the simulation code (e.g., Maeda et al. 2010; Seitenzahl et al. 2013b).

The near- $M_{\mathrm{Ch}}$ model has confronted some obstacles. For example, there are very few observed WDs with masses approaching $M_{\mathrm{Ch}}$ (Giammichele et al. 2012; Kleinman et al. 2013 ), which would be the progenitors for near- $M_{\mathrm{Ch}}$ SNe Ia. ${ }^{7}$

\footnotetext{
$\overline{7}$ However, at least one candidate progenitor for a future near- $M_{\mathrm{Ch}} \mathrm{SN}$ Ia has been discovered (Tang et al. 2014).
} 
Stable accretion to build up a WD to $M_{\mathrm{Ch}}$ imposes very fine requirements on the accretion rate (Shen \& Bildsten 2007). Furthermore, a sufficient number of WDs accreting to produce the SN Ia rate is disfavored by X-ray observations of nearby early-type galaxies (Gilfanov \& Bogdán 2010). Finally, there is a lack of companion stars associated with SN Ia remnants as would be expected in this scenario (e.g., Schaefer \& Pagnotta 2012). One possible solution to these problems is the detonation of a sub- $M_{\mathrm{Ch}}$ WD. Sub- $M_{\mathrm{Ch}}$ WDs are numerous enough to explain SN Ia rates, and their range of masses can explain the range of SN Ia luminosities (Shen et al. 2017).

In addition to the mass of the exploding WD, another outstanding question is the evolutionary path that leads to the explosion. The progenitor systems are generally grouped into "single-degenerate" and "double-degenerate" binaries involving one or more $\mathrm{CO}$ WDs. Both types are possible for near- $M_{\mathrm{Ch}}$ and sub- $M_{\mathrm{Ch}}$ models. The types of explosion can be classified as follows:

1. The original single-degenerate, near- $M_{\mathrm{Ch}}$ model invoked accretion of hydrogen, usually from a red giant, onto a CO WD until it reached $M_{\mathrm{Ch}}$ (e.g., Whelan \& Iben 1973). More recent models (Yoon \& Langer 2003; Brooks et al. 2016) examined the transfer of helium rather than hydrogen. In this case, the $\mathrm{He}$ burns into $\mathrm{C}$ and $\mathrm{O}$ until the WD nears $M_{\mathrm{Ch}}$.

2. The double-degenerate, $M_{\mathrm{Ch}}$ model supposes that two WDs that are individually below $M_{\mathrm{Ch}}$ merge slowly (Webbink 1984). If the merged remnant exceeds $M_{\mathrm{Ch}}$, it will explode. This channel is now disfavored because it is expected to result in accretion-induced collapse into a neutron star (Saio \& Nomoto 1985, 2004; Timmes et al. 1994; Shen et al. 2012).

3. The single-degenerate, sub- $M_{\mathrm{Ch}}$ model is similar to case 1. The difference is that sub- $M_{\mathrm{Ch}}$ WDs can explode if they accrete He slowly enough to accumulate a critical amount before it ignites. The eventual He ignition could be strong enough to shock the inner CO WD to thermonuclear densities and temperatures (Nomoto 1982; Woosley et al. 1986).

4. Double-degenerate, sub- $M_{\mathrm{Ch}}$ explosions are similar to case 2. The difference is in the end phase of the merger. If $\mathrm{He}$ is transferred from the surface of one WD to the other, it could ignite, leading to a detonation (Guillochon et al. 2010; Pakmor et al. 2013; Shen \& Moore 2014; Townsley et al. 2019), similar to case 3. In this case, the secondary WD would survive and fly away at its final orbital velocity of thousands of $\mathrm{km} \mathrm{s}^{-1}$ (Shen et al. 2018a). Alternatively, a violent merger could directly ignite the carbon without the need for $\mathrm{He}$ ignition (Pakmor et al. 2012).

In a variation on case 2, van Kerkwijk et al. (2010) proposed that two WDs can merge smoothly, but compressional heating from the resulting accretion disk can ignite the carbon, even if the final mass does not exceed $M_{\mathrm{Ch}}$.

There is also a separate case of failed explosions in which the WD does not completely disrupt. The idea of a failed explosion is motivated by the existence of Type Iax SNe, a class of sub-luminous SNe (Foley et al. 2013). Kromer et al. (2015) conjectured that Type Iax SNe are weak deflagrations that do not disrupt the WD and that leave behind a remnant. In this scenario, the partially exploded WD would become unbound from its companion. Thus, some hypervelocity WDs might be associated with Type Iax SNe (Vennes et al. 2017; Raddi et al. 2018a, 2018b, 2019) rather than the sub- $M_{\mathrm{Ch}}$, double-degenerate scenario (case 4 above; Shen et al. 2018a). Type Iax SNe likely have a limited effect on galactic chemical evolution because they probably do not eject much mass.

Various individual SNe Ia support nearly all classes of explosion. For example, ultraviolet pulses have been interpreted as the interaction of the SN shock with a red giant companion in SN 2012cg (Marion et al. 2016, rebutted by Shappee et al. 2018), SN 2017cbv (Hosseinzadeh et al. 2017), and iPTF15atg (Cao et al. 2015), which was a peculiar explosion similar to SN 2002es (Ganeshalingam et al. 2012; White et al. 2015). The first two discoveries support the singledegenerate, near- $M_{\mathrm{Ch}}$ model. However, $\mathrm{H} \alpha$ emission is expected in late-time spectroscopy of SNe Ia arising from a H-rich donor. With a few exceptions (i.a., Hamuy et al. 2003; Kollmeier et al. 2019), $\mathrm{H} \alpha$ is rarely found in late-time nebular spectra of SNe Ia (Maguire et al. 2016; Shappee et al. 2018; Tucker et al. 2019). Furthermore, Li et al. (2011) and Bloom et al. (2012) concluded that SN 2011fe did not have a red giant companion. Likewise, the light curve and spectrum of SN 1999 by match a sub- $M_{\mathrm{Ch}}$ detonation but not a $M_{\mathrm{Ch}}$ DDT (Blondin et al. 2018). For similar reasons, the peculiar SN ZTF18aaqeasu seems to have been a double detonation of a sub- $M_{\mathrm{Ch}}$ WD (De et al. 2019). Furthermore, hydrodynamical models of observed SN Ia light curves show that at least some progenitors must be below $M_{\mathrm{Ch}}$ (Goldstein \& Kasen 2018), but with a WD mass function that peaks toward $M_{\mathrm{Ch}}$ (Scalzo et al. 2014). It is important to note that these studies are not necessarily discordant because SNe Ia could explode through multiple channels (e.g., Mannucci et al. 2006). Nonetheless, the purpose of our study is to identify the dominant channel of $\mathrm{SNe}$ Ia in dwarf galaxies.

Nucleosynthesis predictions exist for most of these classes of models. While the nucleosynthesis distinction between singleand double-degenerate models may not be large enough to distinguish with current observational data, the mass of the exploding WD has a large effect on the production of certain elements, such as Mn and Ni (e.g., Seitenzahl et al. 2013a). Therefore, one way to address the nature of $\mathrm{SNe}$ Ia is to measure elemental abundances in SNe Ia, their remnants, the gas that they pollute, and the stars that form from that polluted gas.

It is difficult to measure elemental abundances in the spectra of SNe Ia because the material is optically thick and possibly highly inhomogeneous (Post et al. 2014). Although it is possible to measure the abundances of some elements in SN Ia remnants (e.g., Badenes et al. 2006; Borkowski et al. 2010, 2013; Lopez et al. 2015; Grefenstette et al. 2017; Martínez-Rodríguez et al. 2017), that technique is limited to a small number of very recent explosions, and to the few elements that can be observed with X-ray spectroscopy. One of the more interesting cases is the SN Ia remnant 3C 397, which shows enhancements of Fe-peak elements (Yamaguchi et al. 2015; Dave et al. 2017), suggesting that the progenitor was a near- $M_{\mathrm{Ch}}$ WD.

An alternative method of quantifying SN Ia nucleosynthesis is galactic archaeology. SNe Ia that exploded long ago left a chemical imprint on the surrounding gas and stars. We can compare the amounts of key elements, like $\mathrm{Mn}, \mathrm{Fe}$, and $\mathrm{Ni}$, in those stars to different classes of SN Ia models. The Milky 
Way (MW) is the site of most galactic archaeological studies of $\mathrm{SN}$ physics. It is also common to compare theoretical predictions of certain models to the abundances a single star: the Sun (e.g., Maeda et al. 2010; Seitenzahl et al. 2013a).

Dwarf galaxies provide an attractive alternative to the MW for studying SNe Ia. Dwarf galaxies have simple star formation histories (SFHs), which makes their chemical evolution simple to interpret. The ratio of $\alpha$ elements $(\mathrm{O}, \mathrm{Mg}, \mathrm{Si}$, and others) to $\mathrm{Fe}$ declines steeply with increasing metallicity in dwarf galaxies (Shetrone et al. 2001; Venn et al. 2004; Kirby et al. 2011), which is the signature of a transition from core collapse supernovae (CCSNe) to SNe Ia (e.g., Gilmore \& Wyse 1991). As we will show in Section 3, most of the stars in dwarf galaxies were enriched predominantly by $\mathrm{SNe}$ Ia rather than CCSNe. Furthermore, dwarf galaxies have low metallicity $([\mathrm{Fe} / \mathrm{H}] \lesssim-1)$. As a result, any extra neutrons in the nucleosynthesis can be attributed to the explosive events and simmering rather than metallicity (see Section 4.1).

We present a galactic archaeological approach to identifying the nature of SNe Ia in dwarf galaxies. We use our published catalogs of abundances of $\mathrm{Mg}, \mathrm{Si}, \mathrm{Ca}$, and Fe (Kirby et al. 2010) as well as $\mathrm{Cr}$, Co, and $\mathrm{Ni}$ (Kirby et al. 2018; see Section 2). We fit a simple bi-linear model to the evolution of each element, from which we deduced the CCSN yield (Section 3). Then, we subtract the CCSN yield to isolate the SN Ia yield, which we compare to different classes of SN Ia models (Section 4). Section 5 discusses our conclusions in the context of the broader literature of galactic archaeological studies of SNe Ia, and Section 6 summarizes the paper.

\section{Measurements of Iron-peak Abundances in Dwarf Galaxies}

Kirby et al. (2010) presented a catalog of $\mathrm{Mg}, \mathrm{Si}, \mathrm{Ca}, \mathrm{Ti}$, and Fe abundances for 2961 red giants in eight dwarf spheroidal (dSph) satellites of the MW. Kirby et al. (2018) measured Cr, $\mathrm{Co}$, and $\mathrm{Ni}$ abundances for 1819 of these stars. For the current study, we used a subset of these catalogs to draw inferences about the progenitors of SNe Ia. Here, we give a short recounting of the measurements, which Kirby et al. (2010, 2018) described in detail.

The abundances in the catalog come from spectra obtained with DEIMOS (Faber et al. 2003) on the Keck II telescope. The spectral range was approximately 6300-9100 $\AA$, and the spectral resolution was $\Delta \lambda \sim 1.2 \AA$ (FWHM). The measurements were obtained with spectral synthesis. The syntheses themselves were computed with MOOG (Sneden 1973) under the approximation of local thermodynamic equilibrium (LTE). As described by Kirby et al. (2018), we used the solar abundances of Asplund et al. (2009) except for $\mathrm{Mg}$ and Fe. Instead, we used $12+\log [n(\mathrm{Mg}) / n(\mathrm{H})]=7.58$ and $12+\log [n(\mathrm{Fe}) / n(\mathrm{H})]=7.52$.

We compare these observed abundances to theoretical predictions of the explosions of SNe Ia in Section 4.1. Therefore, it is important to attempt to place the observed abundances on an absolute scale as closely as possible. To this end, Kirby et al. (2018) experimented with non-LTE (NLTE) corrections to the abundances. However, they found that attempts at NLTE corrections actually resulted in decreased abundance accuracy. The accuracy was assessed by computing the dispersion of $\mathrm{Cr}$ and $\mathrm{Co}$ abundances within individual globular clusters, which are not expected to show any such dispersion. Careful attempts to apply NLTE corrections increased the dispersion from the LTE abundances. This outcome likely reflects the method in which the atmospheric parameters were determined because the NLTE corrections usually decrease this dispersion (Bergemann \& Cescutti 2010; Bergemann et al. 2010). Therefore, we used LTE abundances in this work. Kirby et al. (2018) used the globular cluster diagnostic to quantify the systematic error in the abundances, which includes some of the error imposed by assuming LTE. These errors are folded into the present work.

Our catalog contains only measurements with estimated uncertainties less than 0.3 dex. The stars in the catalog pass galaxy membership cuts, described by Kirby et al. (2018), on the basis of surface gravity (inferred from the strength of the Na I 8190 doublet) and radial velocity. In addition to these previous membership criteria, we applied a membership criterion based on Gaia proper motions (Gaia Collaboration et al. 2018). First, we cross-matched our catalog with Gaia. Then, we calculated the mean proper motion in R.A. and decl. weighted by the inverse square of the uncertainties. Finally, we excluded stars that were more than $3 \sigma$ discrepant from this mean value. This procedure excluded between one and five stars for each dSph except Fornax, for which 22 stars were excluded.

We restricted our analysis to seven dSphs. In order of decreasing stellar mass, they are Fornax, Leo I, Sculptor, Leo II, Draco, Sextans, and Ursa Minor. We reserve discussion of Fornax and Leo I until Section 5 because they show qualitatively different evolution of Fe-peak elements from the other five dSphs. As a result, the chemical evolution model we present in Section 3.1 is too simple to capture their abundance distributions. Although Canes Venatici I was part of the Kirby et al. (2010, 2018) catalogs, we do not discuss it at all because its sample size is too small to draw meaningful conclusions.

\section{Trends of Iron-peak Abundances in Dwarf Galaxies}

\subsection{A Simple Model of Chemical Evolution}

The chemical evolution of a galaxy is often expressed as $[\mathrm{X} / \mathrm{Fe}]^{8}$ versus $[\mathrm{Fe} / \mathrm{H}]$, where $[\mathrm{X} / \mathrm{Fe}]$ is the ratio between the abundance of an element $\mathrm{X}$ and the abundance of Fe. The iron abundance, $[\mathrm{Fe} / \mathrm{H}]$, is a proxy for the total abundance of all metals. The expected first-order behavior of $[\mathrm{X} / \mathrm{Fe}]$ is constant at low metallicity but sloped-either positively or negativelyabove some threshold value of $[\mathrm{Fe} / \mathrm{H}]$ (Wheeler et al. 1989; Gilmore \& Wyse 1991).

This expectation is based on the assumption that CCSNe are the only nucleosynthetic sources active at early times. (However, there may be some prompt SNe Ia that violate this assumption, as discussed by Mannucci et al. 2006.) Under the approximation (addressed in Section 3.3) that the CCSN yields are independent of metallicity, $[\mathrm{X} / \mathrm{Fe}]$ will be constant as $[\mathrm{Fe} / \mathrm{H}]$ increases up to a certain threshold metallicity, called $[\mathrm{Fe} / \mathrm{H}]_{\mathrm{Ia}}$. After a delay time, corresponding to $[\mathrm{Fe} / \mathrm{H}]_{\mathrm{Ia}}, \mathrm{SNe} \mathrm{Ia}$ begin to explode. They produce elements in a ratio $[\mathrm{X} / \mathrm{Fe}]$ that may differ from CCSNe. As a result, $[\mathrm{X} / \mathrm{Fe}]$ can begin to change as a function of metallicity at $[\mathrm{Fe} / \mathrm{H}]>[\mathrm{Fe} / \mathrm{H}]_{\text {Ia }}$. The slope will be negative if $\mathrm{SNe}$ Ia produce a smaller ratio of $[\mathrm{X} / \mathrm{Fe}]$ than CCSNe.

Figures 1 through 5 show the evolution of several abundance ratios, $[\mathrm{X} / \mathrm{Fe}]$, as a function of $[\mathrm{Fe} / \mathrm{H}]$ for five of the $\mathrm{dSphs}$ in

\footnotetext{
8 We use "bracket notation": [X/Fe] $=\log \frac{n(\mathrm{X}) / n(\mathrm{Fe})}{n_{\odot}(\mathrm{X}) / n_{\odot}(\mathrm{Fe})}$ where $n$ is atomic number density in the star's atmosphere and $n_{\odot}$ is the number density in the Sun's atmosphere.
} 


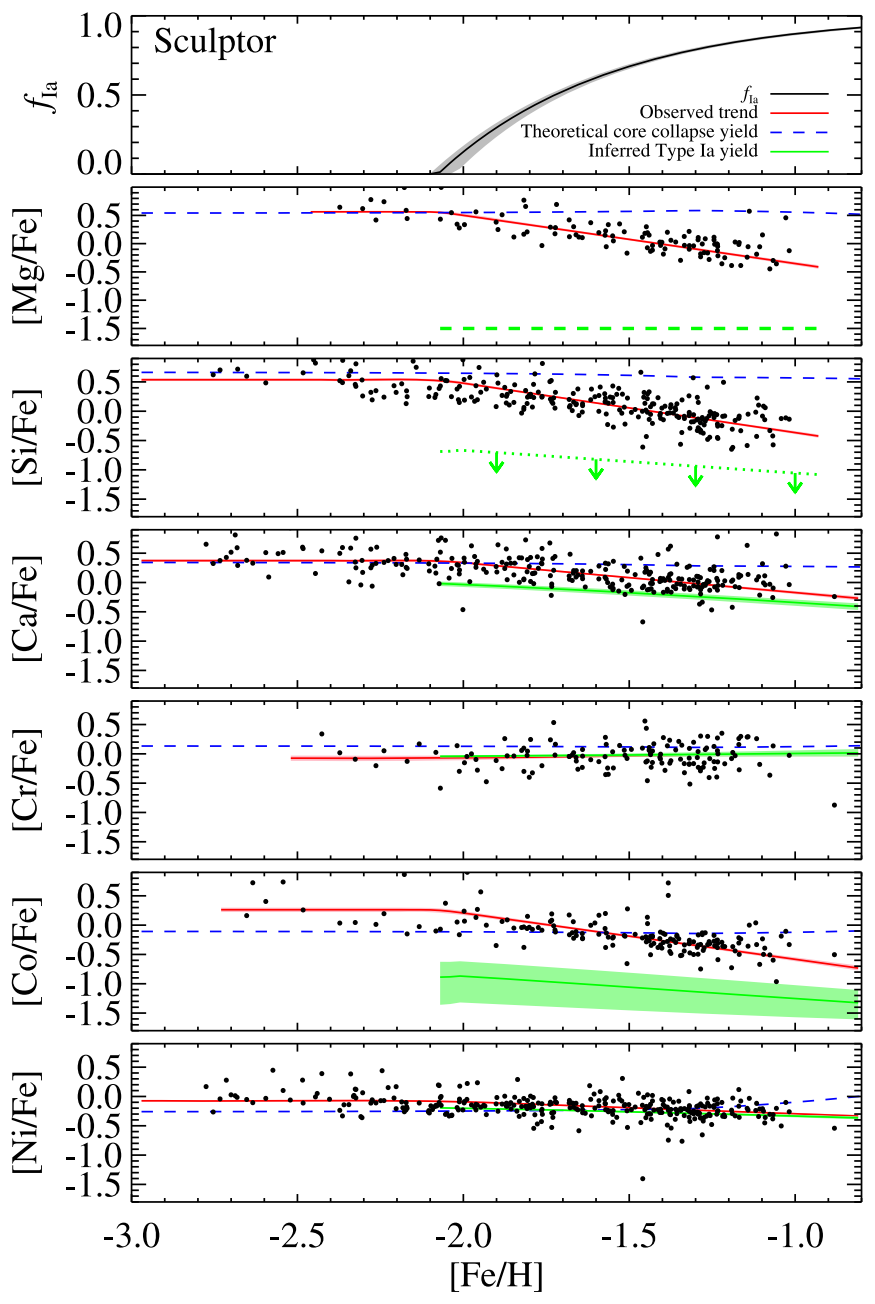

Figure 1. $\mathrm{Mg}, \mathrm{Si}, \mathrm{Ca}, \mathrm{Cr}, \mathrm{Co}$, and $\mathrm{Ni}$ abundance ratios, with respect to $\mathrm{Fe}$, as observed in Sculptor (black points). A simple model (Equation (1), red lines) was fit to the abundances. The $[\mathrm{Mg} / \mathrm{Fe}]$ ratio was used to estimate the fraction of $\mathrm{Fe}$ ( $f_{\text {Ia }}$, top panel) that originated in SNe Ia. Section 3.2 describes a method for isolating the yield ratios (e.g., $[\mathrm{Ni} / \mathrm{Fe}]_{\mathrm{Ia}}$ ) for $\mathrm{SNe} \mathrm{Ia}$, which are shown as green curves. Shaded bands represent the $68 \%$ confidence intervals. Upper limits represent $95 \%$ confidence in cases where the lower bounds of the yields were not well constrained. The dashed blue curves show the metallicity-dependent, initial mass function-averaged yields predicted for CCSNe (Nomoto et al. 2006). The dashed green line in the $\mathrm{Mg}$ panel shows the yield we assumed for SNe Ia.

our sample. The elements (X) are the $\alpha$ elements $\mathrm{Mg}, \mathrm{Si}$, and $\mathrm{Ca}$, and the Fe-peak elements $\mathrm{Cr}, \mathrm{Co}$, and $\mathrm{Ni}$. The behavior of $[\mathrm{X} / \mathrm{Fe}]$ follows the approximate pattern described above. For example, $[\mathrm{Ca} / \mathrm{Fe}]$ in Sculptor (central panel in Figure 1) is flat until $[\mathrm{Fe} / \mathrm{H}] \approx-2.4$ and then declines nearly linearly with increasing $[\mathrm{Fe} / \mathrm{H}]$.

We modeled this behavior as a constant $[\mathrm{X} / \mathrm{Fe}]_{\mathrm{CC}}$ until $[\mathrm{Fe} / \mathrm{H}]_{\mathrm{Ia}}$ followed by a sloped line thereafter. The value of $[\mathrm{X} / \mathrm{Fe}]_{\mathrm{CC}}$ is the result of all of the products of early explosions, including the CCSN yields summed over the initial mass function (IMF) and any prompt SNe Ia. Instead of parameterizing the line by a slope and intercept, we used an angle $(\theta)$ and a perpendicular offset $\left(b_{\perp}\right)$. This allowed us to use a uniform prior on $\theta$, which avoids the preference for shallow slopes when using a uniform prior on the slope (see Hogg et al. 2010).

$$
[\mathrm{X} / \mathrm{Fe}]=\left\{\begin{array}{ll}
{[\mathrm{X} / \mathrm{Fe}]_{\mathrm{CC}}} & \text { if }[\mathrm{Fe} / \mathrm{H}] \leqslant[\mathrm{Fe} / \mathrm{H}]_{\mathrm{Ia}} \\
{[\mathrm{Fe} / \mathrm{H}] \tan \theta+\frac{b_{\perp}}{\cos \theta}} & \text { if }[\mathrm{Fe} / \mathrm{H}]>[\mathrm{Fe} / \mathrm{H}]_{\mathrm{Ia}}
\end{array} .\right.
$$

Enforcing continuity of $[\mathrm{X} / \mathrm{Fe}]$ at $[\mathrm{Fe} / \mathrm{H}]_{\mathrm{Ia}}$ gives the following equation for $b_{\perp}$ in terms of $[\mathrm{X} / \mathrm{Fe}]_{\mathrm{CC}},[\mathrm{Fe} / \mathrm{H}]_{\mathrm{Ia}}$, and $\theta$ :

$$
b_{\perp}=[\mathrm{X} / \mathrm{Fe}]_{\mathrm{CC}} \cos \theta-[\mathrm{Fe} / \mathrm{H}]_{\mathrm{Ia}} \sin \theta .
$$

We fit the chemical evolution in each dSph with Equation (1) using maximum likelihood. There are 13 free parameters for each dSph: $[\mathrm{Fe} / \mathrm{H}]_{\mathrm{Ia}}$ and one pair of $\theta$ and $b_{\perp}$ for each of the six $[\mathrm{X} / \mathrm{Fe}]$ ratios. Following Hogg et al. (2010), the likelihood function is given by the following equations. For a star identified by index $i$ with $[\mathrm{Fe} / \mathrm{H}]_{i} \leqslant[\mathrm{Fe} / \mathrm{H}]_{\text {Ia }}$, the likelihood is relatively simple:

$$
L_{i}=\frac{1}{\sqrt{2 \pi} \delta[\mathrm{X} / \mathrm{Fe}]_{i}} \exp \left(-\frac{\left([\mathrm{X} / \mathrm{Fe}]_{i}-[\mathrm{X} / \mathrm{Fe}]_{\mathrm{CC}}\right)^{2}}{2 \delta[\mathrm{X} / \mathrm{Fe}]_{i}^{2}}\right)
$$

where $\delta[\mathrm{X} / \mathrm{Fe}]_{i}$ represents the measurement uncertainty for star $i$. For a star with $[\mathrm{Fe} / \mathrm{H}]_{i}>[\mathrm{Fe} / \mathrm{H}]_{\mathrm{Ia}}$, the likelihood is

$$
\begin{gathered}
L_{i}=\frac{1}{\sqrt{2 \pi} \Sigma_{i}} \exp \left(-\frac{\Delta_{i}^{2}}{2 \Sigma_{i}^{2}}\right) \\
\Delta_{i}=[\mathrm{X} / \mathrm{Fe}]_{i} \cos \theta-[\mathrm{Fe} / \mathrm{H}]_{i} \sin \theta-b_{\perp} \\
\Sigma_{i}^{2}=\delta[\mathrm{X} / \mathrm{Fe}]_{i}^{2} \cos ^{2} \theta+\delta[\mathrm{Fe} / \mathrm{H}]_{i}^{2} \sin ^{2} \theta .
\end{gathered}
$$

We imposed a prior on $[\mathrm{Mg} / \mathrm{Fe}]_{\mathrm{CC}}$. The purpose of the prior was to ensure that the description of chemical evolution conforms to CCSN yields for Mg. We used predicted yields averaged over the Salpeter (1955) IMF from Nomoto et al. (2006, indicated by a subscripted "N06"). The mathematical form of the prior is

$$
P=\frac{1}{\sqrt{2 \pi} \sigma_{\mathrm{Mg}}} \exp \left(-\frac{\left([\mathrm{Mg} / \mathrm{Fe}]_{\mathrm{N} 06}-[\mathrm{Mg} / \mathrm{Fe}]_{\mathrm{CC}}\right)^{2}}{2 \sigma_{\mathrm{Mg}}^{2}}\right)
$$

The prior, $P$, is multiplied into the likelihood, $L=\prod_{i} L_{i}$. The value of $\sigma_{\mathrm{Mg}}$ sets the strength of the prior. Smaller values lead to a stronger prior, forcing better agreement with the predicted yields. We chose $\sigma_{\mathrm{Mg}}=0.01$, which we consider to be a strong prior (see Section 3.3 and Figure 7).

The fitting method was Markov chain Monte Carlo (MCMC). For computational expediency, we maximized $\ln L$ rather than $L$. The chain had $3.3 \times 10^{5}$ links. The first $3 \times 10^{4}$ links (commonly called "burn-in") were discarded. We imposed uniform priors on all free parameters $\left(\theta\right.$ and $b_{\perp}$ for each element, as well as $[\mathrm{Fe} / \mathrm{H}]_{\mathrm{Ia}}$ ). We imposed an additional prior on a value called $(\mathrm{X} / \mathrm{Fe})_{\mathrm{Ia}}$, described in Section 3.2. The initial value of $[\mathrm{Fe} / \mathrm{H}]_{\text {Ia }}$ was -2.0 , which is approximately the value at which $[\alpha / \mathrm{Fe}]$ begins to decline in most of the $\mathrm{dSphs}$ in our sample. The initial values of $\theta$ and $b_{\perp}$ for each element were given by a simple linear fit to the trend of $[\mathrm{X} / \mathrm{Fe}]$ versus $[\mathrm{Fe} / \mathrm{H}]$ for $[\mathrm{Fe} / \mathrm{H}]>-2.1$. The proposal density for successive links in the chain was based on the Metropolis algorithm. The typical perturbation of $[\mathrm{Fe} / \mathrm{H}]_{\mathrm{Ia}}$ and $b_{\perp}$ was $0.02 \mathrm{dex}$, and the typical perturbation of $\theta$ was $0^{\circ} .2$. The MCMC sampled the posterior distribution of these parameters. We quote the median (50th percentile) value for each quantity. The asymmetric error bars are $68 \%$ confidence intervals around the median.

The best-fit models are shown as red lines in Figures 1 through 5. The pink regions show the $68 \%$ confidence intervals. Table 1 provides the 13 best-fit parameters for each $\mathrm{dSph}$. It also provides the derived quantities $[\mathrm{X} / \mathrm{Fe}]_{\mathrm{Ia}}$ and $[\mathrm{X} / \mathrm{Fe}]_{\mathrm{CC}}$, which are described in Sections 3.2 and 3.3. 
Table 1

Observationally Inferred Yields

\begin{tabular}{|c|c|c|c|c|c|}
\hline Parameter & Scl & Leo II & Dra & Sex & $\mathrm{UMi}$ \\
\hline$\overline{[\mathrm{Fe} / \mathrm{H}]_{\mathrm{Ia}}}$ & $-2.12_{-0.06}^{+0.01}$ & $-1.70_{-0.01}^{+0.00}$ & $-2.36_{-0.06}^{+0.03}$ & $-2.36_{-0.03}^{+0.01}$ & $-2.42_{-0.01}^{+0.00}$ \\
\hline$\theta(\mathrm{Mg})$ & $-42.9_{-0.6}^{+0.4}$ & $-70.4_{-1.4}^{+0.8}$ & $-52.3_{-1.6}^{+0.7}$ & $-63.1_{-1.7}^{+1.1}$ & $-48.9_{-1.7}^{+0.9}$ \\
\hline$b_{\perp}(\mathrm{Mg})$ & $-0.97_{-0.02}^{+0.01}$ & $-1.40_{-0.03}^{+0.02}$ & $-1.45_{-0.05}^{+0.02}$ & $-1.75_{-0.04}^{+0.02}$ & $-1.41_{-0.06}^{+0.03}$ \\
\hline$[\mathrm{Mg} / \mathrm{Fe}]_{\mathrm{CC}}$ & $+0.56 \pm 0.01$ & $+0.56 \pm 0.01$ & $+0.55 \pm 0.01$ & $+0.55 \pm 0.01$ & $+0.55 \pm 0.01$ \\
\hline$\theta(\mathrm{Si})$ & $-42.4_{-0.6}^{+0.4}$ & $-63.6_{-1.1}^{+0.6}$ & $-50.0_{-1.1}^{+0.6}$ & $-59.7_{-1.6}^{+0.8}$ & $-43.4_{-1.2}^{+0.7}$ \\
\hline$b_{\perp}(\mathrm{Si})$ & $-0.97 \pm 0.01$ & $-1.37_{-0.02}^{+0.01}$ & $-1.33_{-0.03}^{+0.02}$ & $-1.56_{-0.03}^{+0.02}$ & $-1.15_{-0.04}^{+0.02}$ \\
\hline$\theta(\mathrm{Ca})$ & $-30.1_{-0.9}^{+0.5}$ & $-48.5_{-1.7}^{+1.0}$ & $-30.2_{-1.8}^{+0.9}$ & $-35.4_{-3.3}^{+2.0}$ & $-37.5_{-2.0}^{+1.0}$ \\
\hline$b_{\perp}(\mathrm{Ca})$ & $-0.69_{-0.02}^{+0.01}$ & $-1.20_{-0.03}^{+0.02}$ & $-0.94_{-0.05}^{+0.03}$ & $-1.10_{-0.09}^{+0.05}$ & $-1.18_{-0.06}^{+0.03}$ \\
\hline$[\mathrm{Ca} / \mathrm{Fe}]_{\mathrm{CC}}$ & $+0.37 \pm 0.02$ & $+0.07 \pm 0.03$ & $+0.20 \pm 0.04$ & $+0.17_{-0.06}^{+0.07}$ & $+0.30 \pm 0.03$ \\
\hline$[\mathrm{Ca} / \mathrm{Fe}]_{\mathrm{Ia}}$ & $-0.17_{-0.05}^{+0.04}$ & $-0.24_{-0.07}^{+0.06}$ & $-0.24 \pm 0.07$ & $-0.22_{-0.09}^{+0.08}$ & $-0.43_{-0.20}^{+0.14}$ \\
\hline$\theta(\mathrm{Cr})$ & $-2.9_{-1.9}^{+1.0}$ & $+48.1_{-4.2}^{+1.8}$ & $-16.9_{-4.3}^{+1.8}$ & $-29.0_{-8.1}^{+3.6}$ & $-43.0_{-1.5}^{+0.9}$ \\
\hline$b_{\perp}(\mathrm{Cr})$ & $-0.11_{-0.05}^{+0.03}$ & $+1.26_{-0.07}^{+0.03}$ & $-0.44_{-0.14}^{+0.06}$ & $-0.87_{-0.21}^{+0.11}$ & $-1.35_{-0.04}^{+0.02}$ \\
\hline$b_{\perp}(\mathrm{Co})$ & $-1.13_{-0.02}^{+0.01}$ & $-1.41_{-0.02}^{+0.01}$ & $-1.43_{-0.04}^{+0.02}$ & $-1.72_{-0.04}^{+0.02}$ & $-1.36_{-0.05}^{+0.02}$ \\
\hline$[\mathrm{Co} / \mathrm{Fe}]_{\mathrm{CC}}$ & $+0.26 \pm 0.04$ & $+0.20 \pm 0.05$ & $+0.32 \pm 0.06$ & $+0.31 \pm 0.12$ & $+0.44 \pm 0.06$ \\
\hline$[\mathrm{Co} / \mathrm{Fe}]_{\mathrm{Ia}}$ & $-1.06_{-0.40}^{+0.25}$ & $-0.58_{-0.32}^{+0.20}$ & $-0.81_{-0.37}^{+0.24}$ & $<-0.60$ & $<-0.47$ \\
\hline$\theta(\mathrm{Ni})$ & $-13.8_{-0.7}^{+0.4}$ & $-24.1_{-4.4}^{+2.4}$ & $-25.0_{-1.8}^{+0.8}$ & $-32.4_{-2.5}^{+1.3}$ & $-32.6_{-1.1}^{+0.6}$ \\
\hline$b_{\perp}(\mathrm{Ni})$ & $-0.55_{-0.02}^{+0.01}$ & $-0.73_{-0.10}^{+0.06}$ & $-1.01_{-0.05}^{+0.02}$ & $-1.20_{-0.06}^{+0.03}$ & $-1.27_{-0.03}^{+0.02}$ \\
\hline$[\mathrm{Ni} / \mathrm{Fe}]_{\mathrm{CC}}$ & $-0.07 \pm 0.01$ & $-0.10_{-0.03}^{+0.04}$ & $-0.08 \pm 0.02$ & $-0.04_{-0.04}^{+0.05}$ & $-0.01 \pm 0.02$ \\
\hline$[\mathrm{Ni} / \mathrm{Fe}]_{\mathrm{Ia}}$ & $-0.26_{-0.02}^{+0.01}$ & $-0.13_{-0.04}^{+0.03}$ & $-0.43_{-0.05}^{+0.04}$ & $-0.44_{-0.06}^{+0.05}$ & $-0.68_{-0.09}^{+0.07}$ \\
\hline
\end{tabular}

Note. All values of $\theta$ are given in degrees. All other values are dex relative to the Sun. The values of $[\mathrm{X} / \mathrm{Fe}]_{\mathrm{Ia}}$ are evaluated at $[\mathrm{Fe} / \mathrm{H}]=-1.5$. Errors represent the $68 \%$ confidence intervals. Asymmetric errors are quoted where the upper and lower errors differ. In cases where $(\mathrm{X} / \mathrm{Fe})_{\mathrm{Ia}}$ was consistent with zero, upper limits on $[\mathrm{X} / \mathrm{Fe}]_{\mathrm{Ia}}$ represent $95 \%$ confidence.

\subsection{Observationally Inferred Yields of SNe Ia}

The amount of an element in a star is a combination of the various nucleosynthetic sources that contributed to its birth cloud. These sources include $\mathrm{SNe}$, winds from intermediateand low-mass stars, and even neutron star mergers. The major contributors to the elements considered in this work $(\mathrm{Mg}, \mathrm{Si}$, $\mathrm{Ca}, \mathrm{Cr}, \mathrm{Fe}, \mathrm{Co}$, and $\mathrm{Ni}$ ) are CCSNe and $\mathrm{SNe} \mathrm{Ia}$.

The amount of an element $\mathrm{X}$ in a star can be represented as a sum of the contribution from both types of $\mathrm{SN}$ :

$$
\mathrm{X}_{*}=\mathrm{X}_{\mathrm{CC}}+\mathrm{X}_{\mathrm{Ia}} \text {. }
$$

We have implicitly assumed that element $\mathrm{X}$ has a negligible contribution from sources other than $\mathrm{SNe}$ and that the abundance of element $\mathrm{X}$ has not changed since the star's birth. We can represent the ratio of two elements in the star as a ratio of their contributions from both types of SNe. Iron is typically used as the comparison element. Bracket notation is not used in the following equations because the element ratios, e.g., $(\mathrm{X} / \mathrm{Fe})$, are linear, not logarithmic:

$$
\left(\frac{\mathrm{X}}{\mathrm{Fe}}\right)_{*}=\frac{\mathrm{X}_{\mathrm{CC}}+\mathrm{X}_{\mathrm{Ia}}}{\mathrm{Fe}_{\mathrm{CC}}+\mathrm{Fe}_{\mathrm{Ia}}} .
$$

We now define a ratio, $R$, of the amount of iron that comes from $\mathrm{SNe}$ Ia compared to the amount that comes from CCSNe.

$$
R \equiv \frac{\mathrm{Fe}_{\mathrm{Ia}}}{\mathrm{Fe}_{\mathrm{CC}}} .
$$

Equation (9) can be expressed in terms of $R$ by dividing the numerator and denominator on the right side by $\mathrm{Fe}_{\mathrm{CC}}$ :

$$
\begin{gathered}
\left(\frac{\mathrm{X}}{\mathrm{Fe}}\right)_{*}=\frac{(\mathrm{X} / \mathrm{Fe})_{\mathrm{CC}}+\mathrm{X}_{\mathrm{Ia}} / \mathrm{Fe}_{\mathrm{CC}}}{1+R} \\
=\frac{(\mathrm{X} / \mathrm{Fe})_{\mathrm{CC}}+R(\mathrm{X} / \mathrm{Fe})_{\mathrm{Ia}}}{1+R} .
\end{gathered}
$$

Equation (12) can then be solved for $R$ :

$$
R=\frac{(\mathrm{X} / \mathrm{Fe})_{\mathrm{CC}}-(\mathrm{X} / \mathrm{Fe})_{*}}{(\mathrm{X} / \mathrm{Fe})_{*}-(\mathrm{X} / \mathrm{Fe})_{\mathrm{Ia}}}
$$

Alternatively, Equation (12) can be solved for $(\mathrm{X} / \mathrm{Fe})_{\mathrm{Ia}}$ :

$$
\left(\frac{\mathrm{X}}{\mathrm{Fe}}\right)_{\mathrm{Ia}}=\frac{R+1}{R}\left(\frac{\mathrm{X}}{\mathrm{Fe}}\right)_{*}-\frac{1}{R}\left(\frac{\mathrm{X}}{\mathrm{Fe}}\right)_{\mathrm{CC}} .
$$

As an aside, we can also write expressions for the fractions of iron that came from SNe Ia $\left(f_{\mathrm{Ia}}\right)$ or $\mathrm{CCSNe}\left(f_{\mathrm{CC}}\right)$ :

$$
\begin{aligned}
f_{\mathrm{Ia}} & \equiv \frac{\mathrm{Fe}_{\mathrm{Ia}}}{\mathrm{Fe}_{\mathrm{CC}}+\mathrm{Fe}_{\mathrm{Ia}}}=\frac{R}{R+1} \\
& =\frac{(\mathrm{X} / \mathrm{Fe})_{\mathrm{CC}}-(\mathrm{X} / \mathrm{Fe})_{*}}{(\mathrm{X} / \mathrm{Fe})_{\mathrm{CC}}-(\mathrm{X} / \mathrm{Fe})_{\mathrm{Ia}}} \\
f_{\mathrm{CC}} & \equiv \frac{\mathrm{Fe}_{\mathrm{CC}}}{\mathrm{Fe}_{\mathrm{CC}}+\mathrm{Fe}_{\mathrm{Ia}}}=\frac{1}{R+1} \\
& =\frac{(\mathrm{X} / \mathrm{Fe})_{*}-(\mathrm{X} / \mathrm{Fe})_{\mathrm{Ia}}}{(\mathrm{X} / \mathrm{Fe})_{\mathrm{CC}}-(\mathrm{X} / \mathrm{Fe})_{\mathrm{Ia}}} .
\end{aligned}
$$


The ratio $R$ is defined only in terms of iron. Thus, Equation (13) can be used to determine $R$ using one element, $\mathrm{X}_{1}$. The resulting value of $R$ is general for any other element, $\mathrm{X}_{2}$, as long as both $\mathrm{X}_{1}$ and $\mathrm{X}_{2}$ can be considered to come exclusively from $\mathrm{SNe}$. Once $R$ is known, Equation (14) can be used to infer the $\mathrm{SN}$ Ia yield for the ratio $(\mathrm{X} / \mathrm{Fe})_{\text {Ia }}$ for any element $\mathrm{X}$.

The ideal element $X_{1}$ for solving Equation (13) is one that has well-known yields from both CCSNe and SNe Ia and is also measured in our spectroscopic sample. Magnesium is the element that best satisfies these criteria; it is synthesized almost exclusively in CCSNe. Nearly all models of SNe Ia agree that virtually no $\mathrm{Mg}$ is produced.

The ratio $R$ can be calculated from $(\mathrm{X} / \mathrm{Fe})_{\mathrm{CC}},(\mathrm{X} / \mathrm{Fe})_{\text {Ia }}$, and $(\mathrm{X} / \mathrm{Fe})_{*}$. We treated the first two quantities as constants, but $(\mathrm{X} / \mathrm{Fe})_{*}$ varies from star to star. In other words, $R$ is a function of time. As in Section 3, we used $[\mathrm{Fe} / \mathrm{H}]$ as a proxy for time because time is not directly observable.

In principle, $R$ could be calculated for individual stars. However, this measurement would be noisy. In some cases, it could even lead to negative (unphysical) values for $R$. Therefore, we used the bi-linear chemical evolution model (Equation (1)) to average over the noise of individual measurements.

The model fits directly gave $[\mathrm{X} / \mathrm{Fe}]_{\mathrm{CC}}$ and $[\mathrm{X} / \mathrm{Fe}]_{*}$ but not $[\mathrm{X} / \mathrm{Fe}]_{\mathrm{Ia}}$. In the case of $\mathrm{Mg}$, we adopted a value of $[\mathrm{Mg} / \mathrm{Fe}]_{\mathrm{Ia}}=-1.5$. For reference, Table 3 provides $[\mathrm{Mg} / \mathrm{Fe}]$ predictions from a variety of SN Ia models. The precise value is not particularly important because the $(\mathrm{Mg} / \mathrm{Fe})_{\mathrm{Ia}}$ ratio in linear units is very close to zero. We have confirmed that changing the value of $[\mathrm{Mg} / \mathrm{Fe}]_{\text {Ia }}$ to -1.0 or -2.5 does not qualitatively alter our inferences of $[\mathrm{X} / \mathrm{Fe}]_{\mathrm{Ia}}$. We even tried $[\mathrm{Mg} / \mathrm{Fe}]_{\mathrm{Ia}}=-0.3$, which is the highest predicted value for the most extreme SN Ia model in Table 3. In this case, some of our lowest inferences of $[\mathrm{X} / \mathrm{Fe}]_{\mathrm{Ia}}$, including $[\mathrm{Si} / \mathrm{Fe}]_{\mathrm{Ia}},[\mathrm{Ca} / \mathrm{Fe}]_{\mathrm{Ia}}$, and $[\mathrm{Co} / \mathrm{Fe}]_{\mathrm{Ia}}$, increased noticeably. Importantly, $[\mathrm{Ni} / \mathrm{Fe}]_{\mathrm{Ia}}$, which is the ratio that most directly affects our conclusions about SNe Ia (Section 4.2), barely changed. Therefore, our results do not depend much on the exact value of $[\mathrm{Mg} / \mathrm{Fe}]_{\mathrm{Ia}}$.

With $[\mathrm{Mg} / \mathrm{Fe}]_{\mathrm{Ia}}$ in hand, we had all of the variables required to solve for $R$ as a function of $[\mathrm{Fe} / \mathrm{H}]$. The top panels of Figures 1 to 5 show $f_{\text {Ia }}=R /(R+1)$ for each of the $\mathrm{dSphs}$ in our sample. The values of $f_{\text {Ia }}$ were calculated at each step in the MCMC chain that evaluated the parameters of the chemical evolution model. The widths of the $f_{\text {Ia }}$ bands in the figures enclose $68 \%$ of the successful MCMC trials.

Once $f_{\text {Ia }}$ (or alternatively, $R$ ) was known as a function of $[\mathrm{Fe} / \mathrm{H}]$ for each $\mathrm{dSph}$, Equation (14) gave the empirical SN Ia yield for an arbitrary element $\mathrm{X}$. We already assumed a value of $[\mathrm{Mg} / \mathrm{Fe}]_{\text {Ia }}$ to calculate $f_{\text {Ia }}$. Therefore, it would not have made sense to use Equation (14) for $\mathrm{Mg}$. On the other hand, we could infer the SN Ia yields of [Si/Fe], $[\mathrm{Ca} / \mathrm{Fe}],[\mathrm{Cr} / \mathrm{Fe}],[\mathrm{Co} / \mathrm{Fe}]$, and $[\mathrm{Ni} / \mathrm{Fe}]$. We did so by evaluating Equation (14) during the MCMC fitting that determined the parameters of the chemical evolution model.

Negative values of $(\mathrm{X} / \mathrm{Fe})_{\text {Ia }}$ are not physical. Therefore, we imposed a prior in the computation of the likelihood function to enforce non-negativity. If an iteration in the MCMC chain yielded one or more negative values of $(\mathrm{X} / \mathrm{Fe})_{\mathrm{Ia}}$, then the likelihood $L$ was made to be zero.

Figures 1 to 5 show in green bands the inferred yield ratios as a function of $[\mathrm{Fe} / \mathrm{H}]$ for each of the $\mathrm{dSphs}$ in our sample.
The widths of the bands reflect the $68 \%$ confidence intervals. Table 1 gives $[\mathrm{X} / \mathrm{Fe}]_{\mathrm{Ia}}$ for each $\mathrm{dSph}$ at a reference metallicity of $[\mathrm{Fe} / \mathrm{H}]=-1.5$.

In some cases, the posterior distributions of $(\mathrm{X} / \mathrm{Fe})_{\text {Ia }}$ are peaked toward zero, i.e., $[\mathrm{X} / \mathrm{Fe}]_{\mathrm{Ia}}$ approaches $-\infty$. These cases can be interpreted as upper limits. All upper limits are quoted at the $95 \%$ confidence level, i.e., the value below which $95 \%$ of the MCMC iterations are found.

Figure 6 shows the inferred $\mathrm{SN}$ Ia yield ratios at $[\mathrm{Fe} / \mathrm{H}]=$ -1.5 for the five dSphs. The inferences for $[\mathrm{Si} / \mathrm{Fe}]_{\text {Ia }}$, $[\mathrm{Ca} / \mathrm{Fe}]_{\mathrm{Ia}}$, and $[\mathrm{Co} / \mathrm{Fe}]_{\mathrm{Ia}}$ are consistent within $2 \sigma$ for all of the dSphs. The formal error bars on $[\mathrm{Cr} / \mathrm{Fe}]_{\mathrm{Ia}}$ and $[\mathrm{Ni} / \mathrm{Fe}]_{\mathrm{Ia}}$ are very small for four of the dSphs, which makes their differences appear especially significant. However, we show in Section 4 that the differences do not complicate our conclusion about the progenitor masses of SNe Ia. Ursa Minor's error bars on $[\mathrm{X} / \mathrm{Fe}]_{\mathrm{Ia}}$ are larger than those of the other dSphs. The size of the error bar results from subtracting a large number from a large number to produce a small number. The fractional error bar (as presented in logarithmic space) is large for this operation. Interestingly, $[\mathrm{Ni} / \mathrm{Fe}]_{\text {Ia }}$ seems to have a significant range among the five $\mathrm{dSphs}$.

We constrained $[\mathrm{Si} / \mathrm{Fe}]_{\mathrm{Ia}}$ to be a small value. The most constraining upper limit, provided by Sculptor, is $[\mathrm{Si} / \mathrm{Fe}]_{\mathrm{Ia}}<$ -0.86 . It may seem odd that we conclude that $\mathrm{SNe}$ Ia make little $\mathrm{Si}$ because they are classified by the presence of $\mathrm{Si}$ absorption in their spectra. However, not much Si production is required to induce deep $\mathrm{Si}$ absorption features in $\mathrm{SNe} \mathrm{Ia}$ spectra. For example, Hachinger et al. (2013) modeled the light curves of the WDD3 and W7 explosion models of Iwamoto et al. (1999). Both models predict $\mathrm{Si}$ and $\mathrm{Fe}$ production at approximately one third the solar ratio $([\mathrm{Si} / \mathrm{Fe}]=-0.5)$. This is more than sufficient to produce theoretical spectra that closely resemble observed spectra of SNe Ia.

\subsection{Comparison to Theoretical Yields of CCSNe}

It is instructive to compare the observationally inferred CCSN yields with theoretical predictions from simulations of CCSNe. Figures 1-5 show the metallicity-dependent predicted CCSN yields of Nomoto et al. (2006) as blue dashed lines. The very slight dependence on metallicity over the metallicity range of our sample justifies our approximation that the CCSN yields are independent of metallicity. Figure 7 compares the CCSN yields inferred from dSphs with the predictions of Nomoto et al. (2006) and Heger \& Woosley (2010). Both studies used one-dimensional hydrodynamical codes, but the explosion initiation was different. Nomoto et al. injected thermal energy (a "thermal bomb"), whereas Heger \& Woosley used a piston (momentum injection). In the case of Nomoto et al., we used the zero-metallicity SN (not hypernova) yields averaged over the Salpeter IMF. Heger \& Woosley presented only zerometallicity yields, but they varied many parameters in the simulations. We used their "standard" explosion model, in which a piston at the base of the oxygen shell initiated the explosion. Mixing followed the "standard" prescription, and the explosion energy was $1.2 \times 10^{51} \mathrm{erg}$. We averaged the yields over a Salpeter IMF in the range $10-100 M_{\odot}$.

The inferred yields generally agree with the predictions. The left panel of Figure 7 shows that the $[\mathrm{Mg} / \mathrm{Fe}]_{\mathrm{CC}}$ inferences fall exactly on the predictions of Nomoto et al. (2006), but we effectively forced this agreement by imposing a strong prior on 


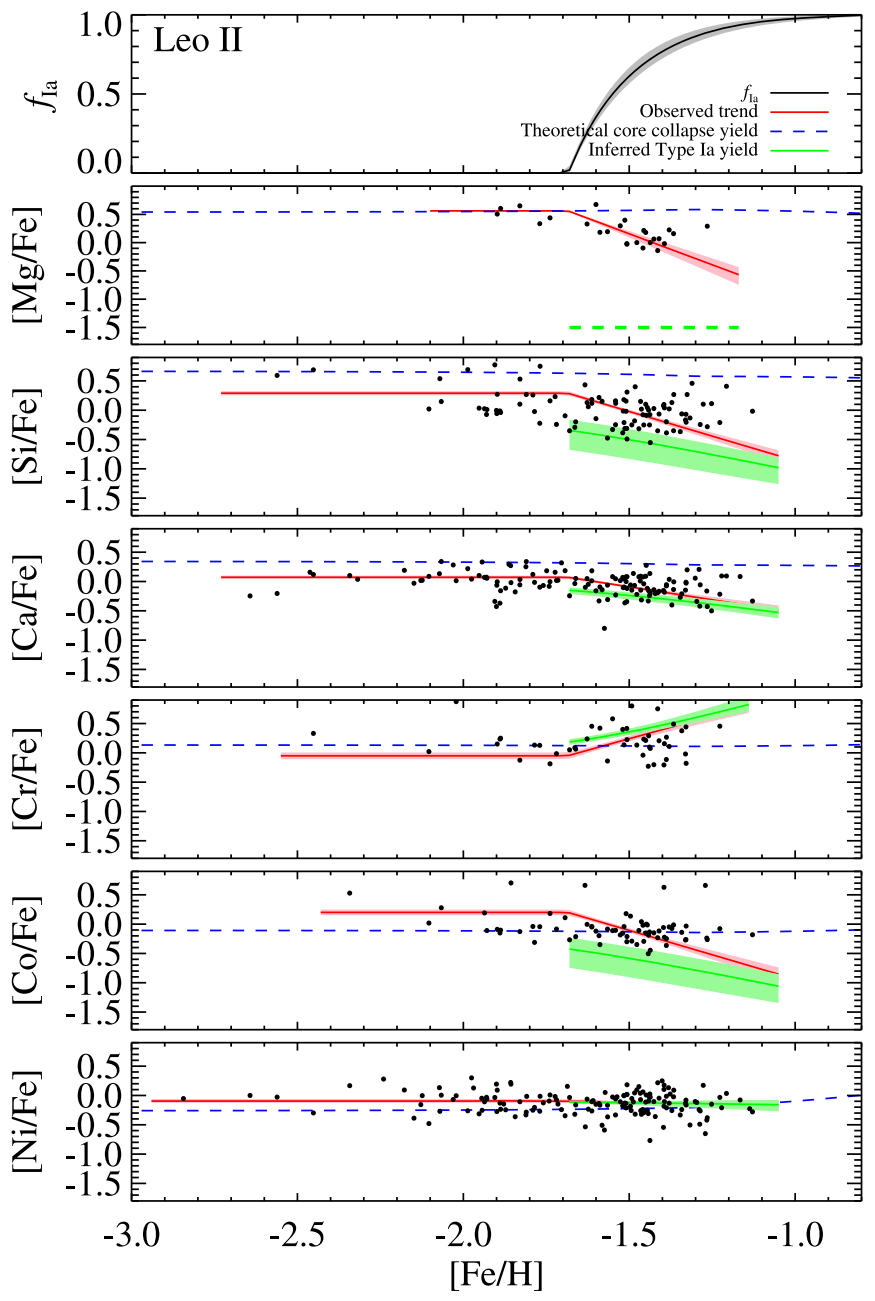

Figure 2. Same as Figure 1 but for Leo II.

$[\mathrm{Mg} / \mathrm{Fe}]_{\mathrm{CC}}$ (Equation (7)). The discrepancy between the two theoretical predictions of $[\mathrm{Mg} / \mathrm{Fe}]_{\mathrm{CC}}$ raises the question whether our choice of prior (Nomoto et al. 2006) affects our conclusions. To address this question, we re-evaluated all of the results of this study using a different prior. First, we note that the observed $[\mathrm{Mg} / \mathrm{Fe}]$ ratios in the stars reach as high as $[\mathrm{Mg} / \mathrm{Fe}]_{\text {N06. }}$. Therefore, it would not make sense to consider a lower value of $[\mathrm{Mg} / \mathrm{Fe}]_{\mathrm{CC}}$. Instead, we considered $[\mathrm{Mg} / \mathrm{Fe}]_{\mathrm{CC}}=+0.8$, roughly in line with Heger \& Woosley's (2010) prediction. While the value of $[\mathrm{Fe} / \mathrm{H}]_{\mathrm{Ia}}$ did tend to decrease when assuming a larger prior on $[\mathrm{Mg} / \mathrm{Fe}]_{\mathrm{CC}}$, the conclusions we draw in Section 4 did not change. In particular, the results shown in Figure 6 shifted by the size of the error bars or less. Therefore, our conclusions on the progenitors of $\mathrm{SNe}$ Ia are mostly insensitive to reasonable choices of the $[\mathrm{Mg} / \mathrm{Fe}]_{\mathrm{CC}}$ prior.

For most galaxies, the inferences for $[\mathrm{Si} / \mathrm{Fe}]_{\mathrm{CC}}$ agree well with both sets of yields, whereas the inferences for $[\mathrm{Ca} / \mathrm{Fe}]_{\mathrm{CC}}$ scatter around Nomoto et al.'s predictions, and the inferences for $[\mathrm{Cr} / \mathrm{Fe}]_{\mathrm{CC}}$ scatter on either side of both sets of predictions. Leo II is an outlier in $[\mathrm{Si} / \mathrm{Fe}]_{\mathrm{CC}}$ and $[\mathrm{Ca} / \mathrm{Fe}]_{\mathrm{CC}}$. It is possible that our bi-linear model is not as well suited to Leo II's more extended SFH compared to the other dSphs. The inferences for $[\mathrm{Co} / \mathrm{Fe}]_{\mathrm{CC}}$ exceed the predictions of both Nomoto et al. and Heger \& Woosley by several tenths of a dex. This discrepancy could indicate that $\mathrm{CCSNe}$ are more neutron-rich than the

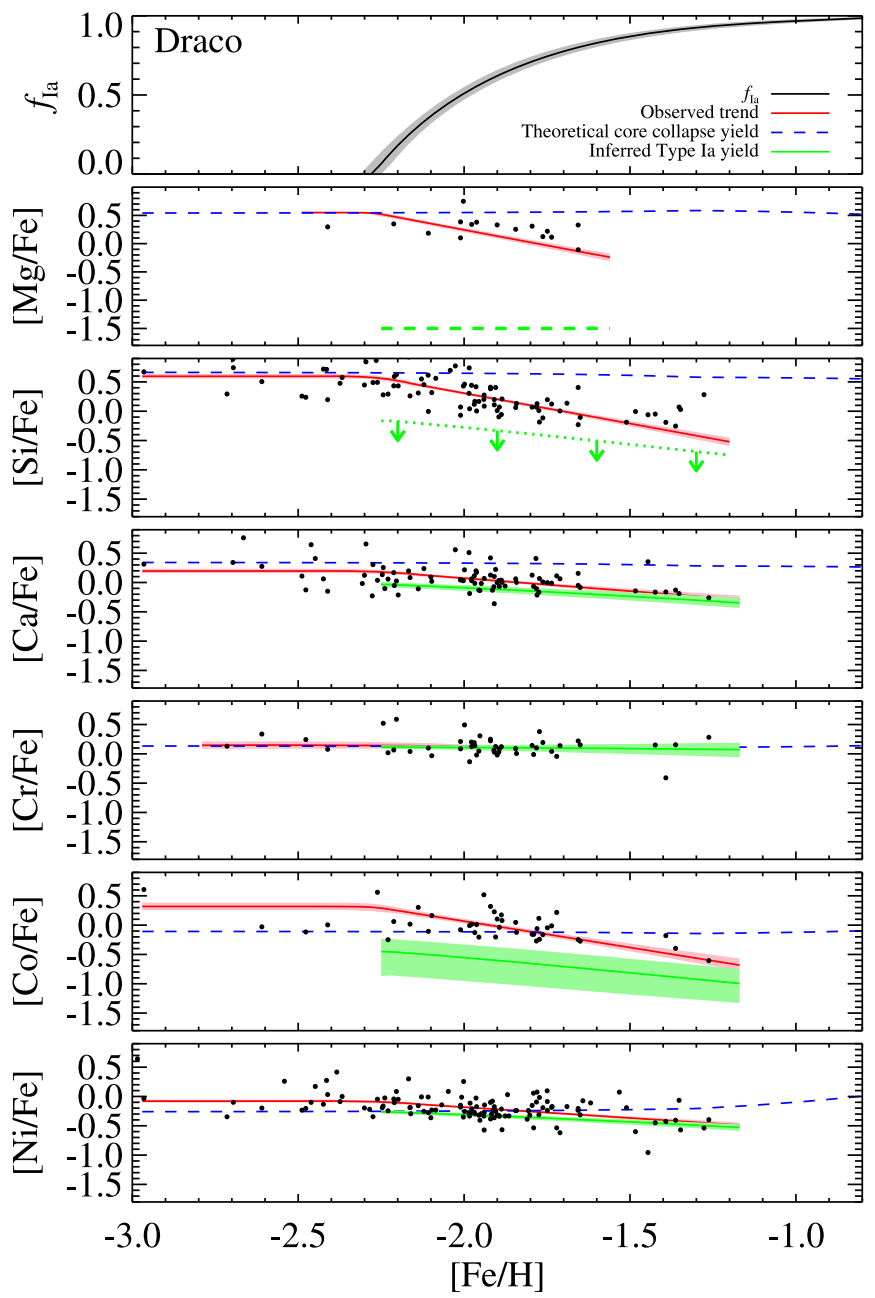

Figure 3. Same as Figure 1 but for Draco.

simulations assumed, or it could indicate that we have not properly accounted for NLTE corrections for the Co abundance measurements (see Kirby et al. 2018). Interestingly, the inferences for $[\mathrm{Ni} / \mathrm{Fe}]_{\mathrm{CC}}$ agree well with those of Heger \& Woosley but less well with those of Nomoto et al.

\subsection{Discussion of the Model}

The "kinked line" description of chemical evolution in Section 3.1 is simplistic. First, the chemical evolution depends sensitively on the star formation rate (SFR). Any increase in SFR, such as a revived burst of star formation, will be accompanied by an increase in the ratio of CCSNe to SNe Ia, which will bring $[\mathrm{X} / \mathrm{Fe}]$ closer to the CCSN value. Therefore, the details of the shape-including deviations from a straight line - of $[\mathrm{X} / \mathrm{Fe}]$ versus $[\mathrm{Fe} / \mathrm{H}]$ can be predicted only if the $\mathrm{SFH}$ is known. Second, the model assumes that $\mathrm{SNe}$ Ia have an appreciable delay time. However, empirically derived delay time distributions indicate that the minimum delay is much less than $420 \mathrm{Myr}$ and possibly as small as $40 \mathrm{Myr}$ (Maoz et al. 2012; Maoz \& Graur 2017). As a result, the period of chemical evolution corresponding to CCSN-only nucleosynthesis may be so short as to be irrelevant. In this case, any changes in the slope of $[\mathrm{X} / \mathrm{Fe}]$ with $[\mathrm{Fe} / \mathrm{H}]$ do not indicate the onset of SNe Ia but rather a change in the SFR. Third, both CCSN and SN Ia yields for some elements depend on the progenitor's metallicity (Woosley \& Weaver 1995; Nomoto et al. 2006; 


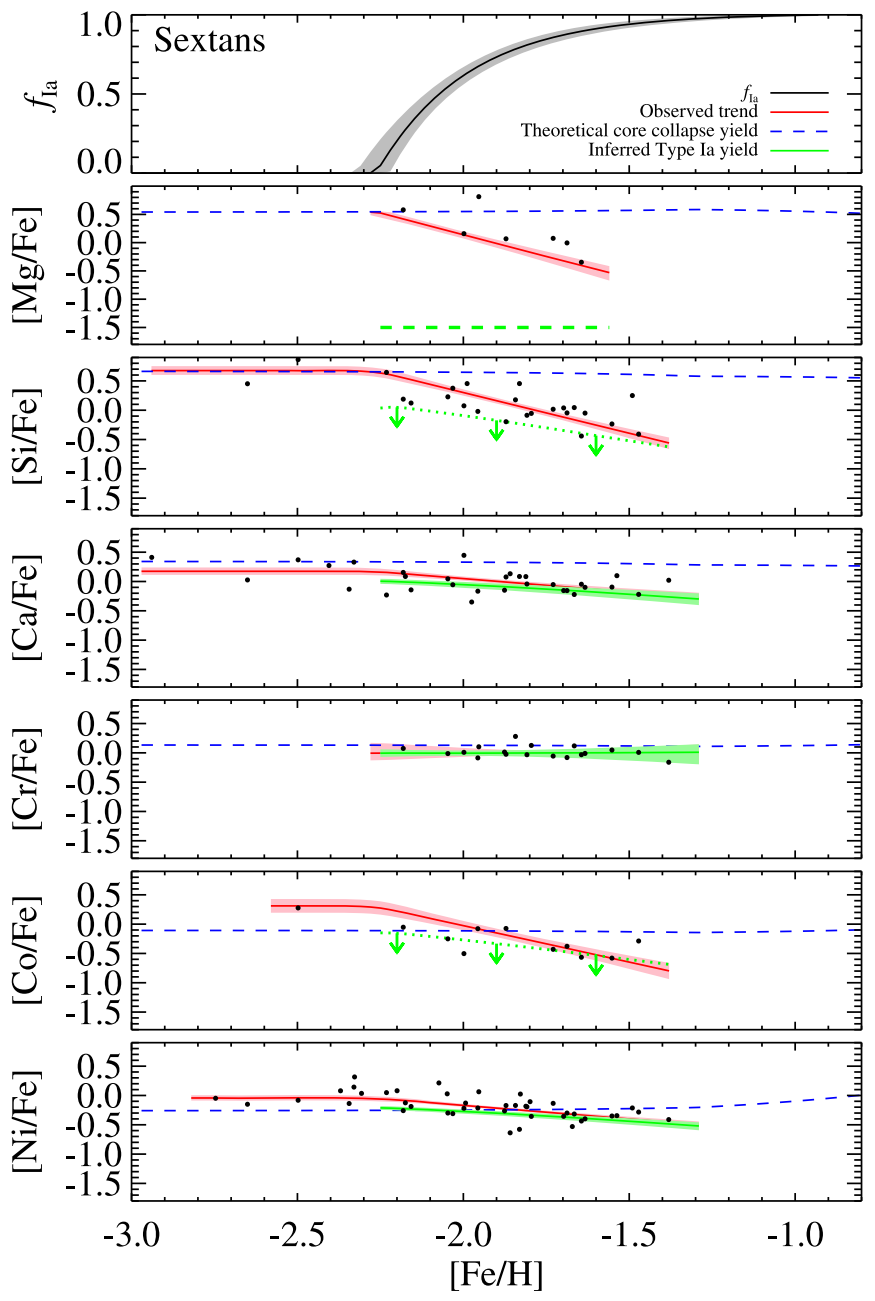

Figure 4. Same as Figure 1 but for Sextans.

Piersanti et al. 2017). As a result, $[\mathrm{X} / \mathrm{Fe}]$ may not be constant at low $[\mathrm{Fe} / \mathrm{H}]$, even if there is a period of chemical evolution from CCSNe only. (However, the yields predicted by Nomoto et al. 2006 have a negligible dependence on metallicity over the metallicity range of our sample, as discussed in Section 3.3.) Fourth, the yield of a stellar population depends on the stellar IMF. Consequently, $[\mathrm{X} / \mathrm{Fe}]$ depends not only on SFR but also on the IMF. Finally, the SN Ia yields could change with time. For example, if $\mathrm{SNe}$ Ia explode at masses below $M_{\mathrm{Ch}}$, the typical mass of exploding WDs could decrease over time as WDs of lower mass begin to appear (e.g., Shen et al. 2017).

Despite its simplicity, the "kinked line" model of chemical evolution still provides a good estimate of the ratio of CCSNe to SNe Ia, provided that the galaxy's gas is well mixed at all times. Kirby et al. (2011) and Escala et al. (2018) showed that this assumption is reasonable for ancient dwarf galaxies (smaller than Fornax) by demonstrating that $[\alpha / \mathrm{Fe}]$ has little dispersion at a given $[\mathrm{Fe} / \mathrm{H}]$. As long as $[\mathrm{Fe} / \mathrm{H}]$ can be used as a proxy for time, then the small dispersion in $[\alpha / \mathrm{Fe}]$ indicates that the mixing timescale is shorter than the star formation timescale. As a result, none of the shortcomings of the "kinked line" model enumerated in the previous paragraph leads to any ambiguity in interpreting $[\mathrm{X} / \mathrm{Fe}]$ as a linear combination of $\mathrm{CCSNe}$ and SNe Ia. We do need to assume that the elements
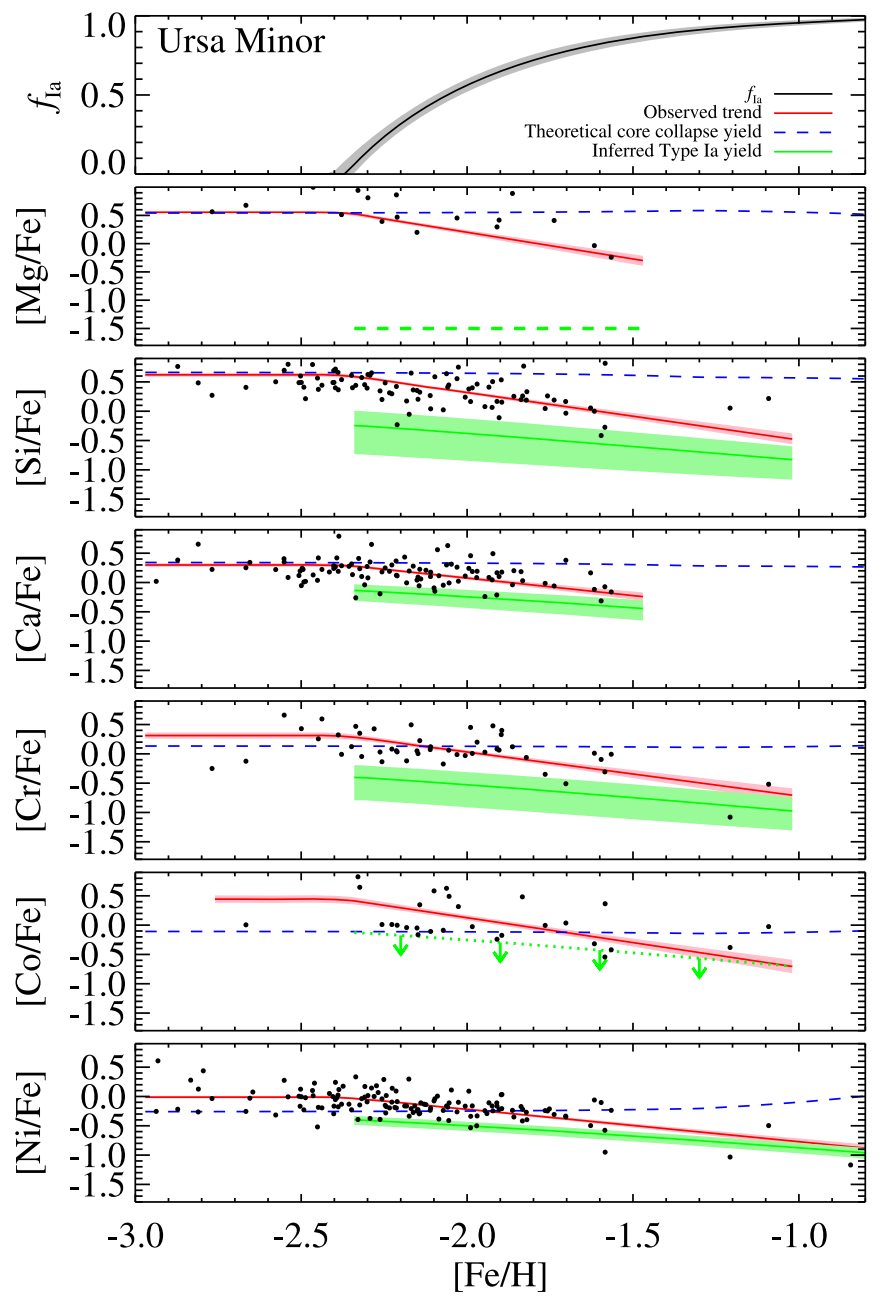

Figure 5. Same as Figure 1 but for Ursa Minor.

considered here originate exclusively in CCSNe and SNe Ia, which we believe to be a good assumption.

\subsection{Comparison to Prior Work}

In nearly all of the cases studied here, the elemental ratios decline with increasing metallicity. This behavior indicates that Fe-peak elements are produced more slowly relative to $\mathrm{Fe}$ as the dwarf galaxies evolve.

Previous studies have noted the same pattern in other dwarf galaxies. For example, Cohen \& Huang (2010) demonstrated with high-resolution spectra that $[\mathrm{Co} / \mathrm{Fe}]$ and $[\mathrm{Ni} / \mathrm{Fe}]$ decline with increasing $[\mathrm{Fe} / \mathrm{H}]$ in Ursa Minor. On the other hand, they found the two lowest-metallicity stars in their sample had $[\mathrm{Cr} / \mathrm{Fe}]$ ratios significantly lower than the other stars. Although these two stars are in our sample, the signal-to-noise ratio of the DEIMOS spectra is not sufficient to measure any Fe-peak abundance other than $\mathrm{Ni}$. The trend of $[\mathrm{Cr} / \mathrm{Fe}]$ for the stars with $-2.5<[\mathrm{Fe} / \mathrm{H}]<-1.5$ is approximately flat in both samples.

The decline of $[\mathrm{Co} / \mathrm{Fe}]$ and $[\mathrm{Ni} / \mathrm{Fe}]$ with increasing $[\mathrm{Fe} / \mathrm{H}]$ has also been noted in more massive galaxies, such as Fornax (Letarte et al. 2010; Lemasle et al. 2014) and Sagittarius (Sbordone et al. 2007; Hasselquist et al. 2017). After NLTE corrections, Bergemann et al. (2010) and Bergemann \& Cescutti (2010) also measured near-solar ratios of $[\mathrm{Cr} / \mathrm{Fe}]$ 


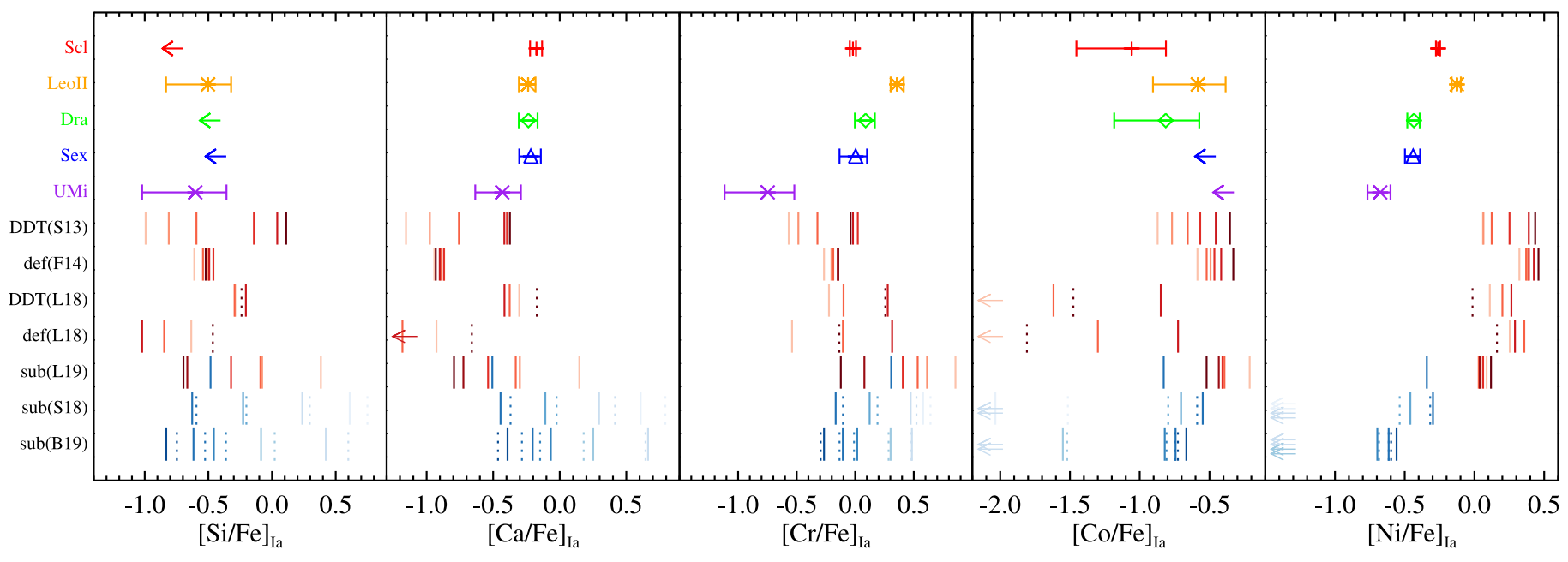

Figure 6. SN Ia yields at $[\mathrm{Fe} / \mathrm{H}]=-1.5$ inferred from observations of dSph stars (rainbow colors) compared to theoretically predicted yields for various models (described in Table 2). The dSphs are ordered from most massive (Sculptor) to least massive (Ursa Minor). For the theoretical yields, shades of red indicate yields at solar metallicity, whereas shades of blue indicate a metallicity of $10^{-1.5} Z_{\odot}$. Lighter shading indicates fewer ignition sites (S13 and F14), lower initial density (L18), or lower-mass WDs (L19, S18, and B19). Dotted lines represent special cases: WDD2 or W7 (L18), C/O = 30/70 (S18), or $\xi_{\mathrm{CO}}=0.0$ (B19). Leftward-pointing arrows for the theoretical yields indicate yield ratios below the left limit of the plot.

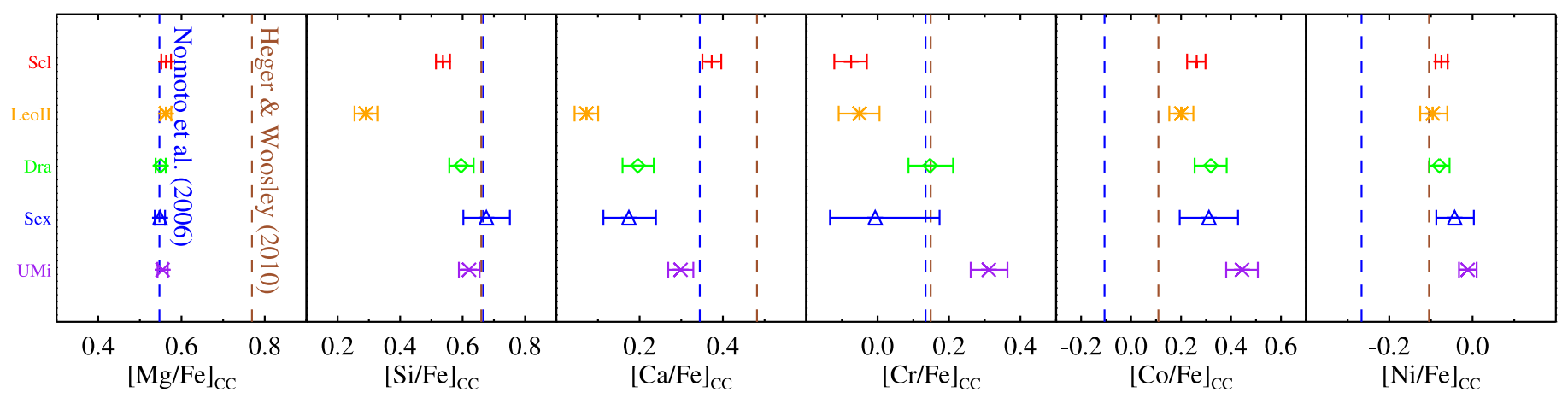

Figure 7. CCSN yields inferred from observations of dSph stars compared to theoretically predicted yields (Nomoto et al. 2006, dashed blue line, and Heger \& Woosley 2010, dashed brown line).

and declining $[\mathrm{Co} / \mathrm{Fe}]$ with increasing metallicity in the metalpoor Galactic halo. Although the Large Magellanic Cloud does not seem to display these decreasing iron-peak-to-iron ratios, the ratios are sub-solar (Pompéia et al. 2008). This behavior is not limited to Co and Ni. Even $\mathrm{Zn}(Z=30$, not measurable in our spectra) shows a downward trend of $[\mathrm{Zn} / \mathrm{Fe}]$ with increasing $[\mathrm{Fe} / \mathrm{H}]$ in Sculptor and other Local Group dwarf galaxies (Skúladóttir et al. 2017).

Taken as a whole, the $[\mathrm{Co} / \mathrm{Fe}]$ and $[\mathrm{Ni} / \mathrm{Fe}]$ ratios of the MW's dwarf galaxies seem to follow the same pattern as $[\alpha / \mathrm{Fe}]$. The ratios are lower than in the MW at the same metallicity, and they decline with metallicity in nearly all cases. The interpretation in the context of the above description of chemical evolution is that dwarf galaxies experienced a larger ratio of SNe Ia:CCSNe than the components of the MW at comparable metallicity (the MW halo for smaller dwarf galaxies and the MW disks for the Magellanic Clouds). Furthermore, the average SN Ia in dwarf galaxies produces stable $\mathrm{Co}$ and $\mathrm{Ni}$ in a lower proportion relative to $\mathrm{Fe}$ than the average CCSN.

\section{Constraints on SNe Ia}

The $[\alpha / \mathrm{Fe}]$ ratios in dwarf galaxies indicate that their chemical evolution is dominated by SNe Ia at late times. This conclusion can be drawn from the low values of $[\mathrm{O} / \mathrm{Fe}]$ and $[\mathrm{Mg} / \mathrm{Fe}]$, among other ratios, found in the stars with higher metallicities in the dwarf galaxies (Shetrone et al. 2001, 2003; Venn et al. 2004; Kirby et al. 2011). The dominance of SNe Ia makes dwarf galaxies among the best places to study the effect of SNe Ia on chemical evolution. However, many dSphs, including the five main galaxies in this paper, have short SFHs. As a result, an individual dSph with a specific SFH may not sample all varieties of SNe Ia, some of which could have delay times exceeding several Gyr.

In Section 3.2, we used the $[\mathrm{Mg} / \mathrm{Fe}]$ ratio to estimate the amount of $\mathrm{Fe}$ that comes from CCSNe versus SNe Ia in each star. We subtracted the CCSN contribution from the observed abundance ratio of each Fe-peak element to estimate the ratio from $\mathrm{SNe}$ Ia only. We now compare these observationally inferred SN Ia yields to theoretical predictions of SN Ia yields.

\subsection{Theoretical Yields of SNe Ia}

Several nucleosynthetic predictions have been published in the last several years for different types of SN Ia explosions. We considered six recent studies that sampled a variety of explosion types.

Table 2 summarizes the salient features of the explosion simulations, which we describe in more detail below. The table 
Table 2

Type Ia Supernova Models

\begin{tabular}{lll}
\hline \hline Model & Authors & Description \\
\hline DDT(S13) & Seitenzahl et al. (2013b) & $M_{\mathrm{Ch}}$, 3D, DDT, multiple ignition sites \\
$\operatorname{def(F14)~}$ & Fink et al. (2014) & $M_{\mathrm{Ch}}$, 3D, pure deflagration, multiple ignition sites \\
DDT(L18) & Leung \& Nomoto (2018) & $M_{\mathrm{Ch}}$, 2D, DDT, varying initial central density \\
$\operatorname{def(L18)}$ & Leung \& Nomoto (2018) & $M_{\mathrm{Ch}}, 2 \mathrm{D}$, pure deflagration, varying initial central density \\
sub(L19) & Leung \& Nomoto (2019) & sub- $M_{\mathrm{Ch}}, 2 \mathrm{D}$, double detonation with He shell \\
sub(S18) & Sub- $M_{\mathrm{Ch}}$, 1D, detonation of bare CO WD, two choices of C/O mass ratio \\
sub(B19) & Bravo et al. (2018b) & sub- $M_{\mathrm{Ch}}, 1 \mathrm{D}$, detonation of bare CO WD, two choices of ${ }^{12} \mathrm{C}+{ }^{16} \mathrm{O}$ reaction rate \\
\hline
\end{tabular}

assigns abbreviations to the studies: "DDT," pure deflagration ("def"), and sub-Chandrasekhar detonations ("sub"). The code in parentheses refers to the first author and year of publication. Leung \& Nomoto (2018, L18) simulated both DDTs and pure deflagrations. Each of the models varied some parameters that affected the nucleosynthesis. We considered a subset of those models, focusing on the models that their authors considered "standard" or "benchmark" models. Table 3 gives the [Si/Fe], $[\mathrm{Ca} / \mathrm{Fe}],[\mathrm{Cr} / \mathrm{Fe}],[\mathrm{Co} / \mathrm{Fe}]$, and $[\mathrm{Ni} / \mathrm{Fe}]$ yield predictions from each those models. Figure 6 also represents the theoretical yields as vertical lines.

DDT: We chose two sets of near- $M_{\mathrm{Ch}}$ DDT models. Multidimensional simulations are particularly important for deflagrations, where the burning front is highly textured. Therefore, we considered only multi-dimensional deflagration simulations. (However, Katz \& Zingale 2019 cautioned that all multidimensional SN Ia simulations to date probably have not resolved the initiation of detonation.)

Seitenzahl et al. (2013b, S13) computed 3D models with multiple off-center ignition sites in CO WDs with a central density of $\rho_{c}=2.9 \times 10^{9} \mathrm{~g} \mathrm{~cm}^{-3}$. The model names specify the number of ignition sites. For example, N100 (the original model of Röpke et al. 2012) has 100 ignition sites. Most models assume that the WD is $47.5 \%{ }^{12} \mathrm{C}, 50 \%{ }^{16} \mathrm{O}$, and $2.5 \%$ ${ }^{22} \mathrm{Ne}$ by mass. The amount of ${ }^{22} \mathrm{Ne}$ corresponds to the amount expected in a WD after the evolution of a solar-metallicity star. S13 also considered lower metallicity versions of the N100 model, which we used in our work. They also constructed models of low density, high density, and a compact configuration of ignition sites, but we did not consider them.

We also considered the DDT models of L18. In contrast to the S13 models, the L18 models were computed in 2D, and the detonation began at a single point in the center of the WD. L18 simulated a variety of central densities. We considered the models at $\rho_{c}=\{1,3,5\} \times 10^{9} \mathrm{~g} \mathrm{~cm}^{-3}$. They used the same initial composition as S13. They also treated metallicity in the way described above, but they considered a range of metallicity for most of their models. Finally, L18 updated "WDD2," the classic DDT model of Iwamoto et al. (1999), with modern electron capture rates. We also considered that updated model.

Pure deflagrations: Our comparison set also includes two sets of pure deflagrations of near- $M_{\mathrm{Ch}}$ WDs. These models are often considered to represent Type Iax SNe (e.g., Kromer et al. 2015). As a result, the simulated properties, like the light curve, spectrum, and nucleosynthesis yields, may not be applicable to "normal" SNe Ia.

Fink et al. (2014, F14) based their 3D simulations of pure deflagrations closely on the DDT models of S13. The F14 models also presumed between 1 and 1600 off-center sites of ignition, but they did not transition to detonations. They considered only solar metallicity.

L18 also computed pure deflagrations in addition to their DDT models. The deflagration and DDT models were exploded with the same initial values of central density and metallicity. As they did with the WDD2 model, L18 also updated the pure-deflagration "W7" model of Iwamoto et al. (1999). We included that model in our comparison set. W7 is the only deflagration model considered here that is computed at a variety of non-solar metallicities.

Sub- $M_{\mathrm{Ch}}$ : We also compared our inferred yields against three sets of sub- $M_{\mathrm{Ch}}$ detonations. Leung \& Nomoto (2019, L19) used the same 2D code as their earlier work. They exploded the simulated WDs using double detonation. The WDs were equal parts $\mathrm{C}$ and $\mathrm{O}$ by mass. The first detonation started in a He shell on the surface. They simulated various $\mathrm{He}$ shell masses $\left(M_{\mathrm{He}}\right)$. The He detonation shocked the interior $\mathrm{C}$ and $\mathrm{O}$, which caused a second detonation. They considered WD masses in the range 0.90 to $1.20 M_{\odot}$. All models were computed at solar metallicity except the $1.10 \mathrm{M}_{\odot}$ ("benchmark") model, which was also computed at metallicities ranging from 0.1 to $5 Z_{\odot}$. Metallicity was approximated by the amount of ${ }^{22} \mathrm{Ne}$ present.

Shen et al. (2018a, S18) simulated 1D, spherically symmetric detonations of bare CO WDs. The detonations began at the centers of the WDs. They explored the effect of the $\mathrm{C} / \mathrm{O}$ ratio on the nucleosynthesis by simulating both $\mathrm{C} / \mathrm{O}=50 / 50$ and 30/70, which is more representative of the ratio expected in actual WDs. They considered masses from 0.8 to $1.1 M_{\odot}$ and metallicities from 0 to $2 Z_{\odot}$.

Bravo et al. (2019, B19) also conducted 1D simulations of detonations that began at the centers of sub- $M_{\mathrm{Ch}}$ WDs. ${ }^{9}$ They treated composition and metallicity in the same manner as L19. They also explored the effect of reducing the reaction rate of ${ }^{12} \mathrm{C}+{ }^{16} \mathrm{O}$ by a factor of 10 . The models with the reduced reaction rate are represented by $\xi_{\mathrm{CO}}=0.9$ in Table 3 . The models with the "standard" reaction rate have $\xi_{\mathrm{CO}}=0.0$.

We first discuss differences between the models before comparing the observations to theoretical predictions. First, the metallicity of the WD influences the yields, sometimes by a large amount. Timmes et al. (2003) studied how the ${ }^{22} \mathrm{Ne}$ content, which depends directly on initial metallicity, affects neutronization in the WD core. Piro \& Bildsten (2008) and Chamulak et al. (2008) further studied pre-explosion "simmering," or convective burning in the core prior to explosion, in near- $M_{\mathrm{Ch}} \mathrm{SN}$ Ia progenitors. The effect of simmering is to increase the neutron excess. In effect, it makes the initial metallicity of a $M_{\mathrm{Ch}}$ SN Ia irrelevant below a threshold

\footnotetext{
9 B19 also simulated DDT explosions, but we did not consider them because we only considered multi-dimensional simulations of deflagrations.
} 
Table 3

Theoretically Predicted Yields

\begin{tabular}{|c|c|c|c|c|c|c|c|}
\hline Model & $\log \left(Z / Z_{\odot}\right)$ & {$[\mathrm{Mg} / \mathrm{Fe}]$} & {$[\mathrm{Si} / \mathrm{Fe}]$} & {$[\mathrm{Ca} / \mathrm{Fe}]$} & {$[\mathrm{Cr} / \mathrm{Fe}]$} & {$[\mathrm{Co} / \mathrm{Fe}]$} & {$[\mathrm{Ni} / \mathrm{Fe}$} \\
\hline \multicolumn{8}{|c|}{ DDT(S13) } \\
\hline $\mathrm{N} 1$ & 0.0 & -2.16 & -0.99 & -1.16 & -0.57 & -0.87 & +0.06 \\
\hline N3 & 0.0 & -1.87 & -0.81 & -0.98 & -0.49 & -0.77 & +0.06 \\
\hline N10 & 0.0 & -1.81 & -0.59 & -0.76 & -0.32 & -0.66 & +0.12 \\
\hline N100 & 0.0 & -1.38 & -0.14 & -0.40 & +0.02 & -0.57 & +0.25 \\
\hline N200 & 0.0 & -0.92 & +0.04 & -0.42 & -0.02 & -0.45 & +0.39 \\
\hline N1600 & 0.0 & -0.92 & +0.11 & -0.37 & -0.04 & -0.35 & +0.44 \\
\hline \multicolumn{8}{|c|}{$\operatorname{def}(\mathrm{F} 14)$} \\
\hline N1def & 0.0 & -1.30 & -0.61 & -0.94 & -0.27 & -0.59 & +0.32 \\
\hline N3def & 0.0 & -1.16 & -0.50 & -0.87 & -0.20 & -0.49 & +0.38 \\
\hline N10def & 0.0 & -1.25 & -0.54 & -0.89 & -0.19 & -0.52 & +0.37 \\
\hline N100def & 0.0 & -1.01 & -0.46 & -0.87 & -0.15 & -0.46 & +0.39 \\
\hline N200def & 0.0 & -1.09 & -0.49 & -0.90 & -0.15 & -0.42 & +0.43 \\
\hline N1600def & 0.0 & -1.11 & -0.52 & -0.93 & -0.15 & -0.33 & +0.46 \\
\hline \multicolumn{8}{|c|}{ DDT(L18) } \\
\hline WDD2 & 0.0 & -1.73 & -0.24 & -0.17 & +0.26 & -1.48 & -0.01 \\
\hline DDT $1 \times 10^{9} \mathrm{~g} \mathrm{~cm}^{-3}$ & 0.0 & -2.36 & -0.29 & -0.30 & -0.22 & -4.26 & +0.11 \\
\hline DDT $3 \times 10^{9} \mathrm{~g} \mathrm{~cm}^{-3}$ & 0.0 & -2.59 & -0.29 & -0.38 & -0.10 & -1.62 & +0.20 \\
\hline DDT $5 \times 10^{9} \mathrm{~g} \mathrm{~cm}^{-3}$ & 0.0 & -2.53 & -0.21 & -0.42 & +0.28 & -0.85 & +0.26 \\
\hline \multicolumn{8}{|c|}{$\operatorname{def}(\mathrm{L} 18)$} \\
\hline W7 & 0.0 & -2.01 & -0.46 & -0.66 & -0.14 & -1.81 & +0.16 \\
\hline def $1 \times 10^{9} \mathrm{~g} \mathrm{~cm}^{-3}$ & 0.0 & -1.59 & -0.63 & -0.93 & -0.54 & -4.09 & +0.25 \\
\hline def $3 \times 10^{9} \mathrm{~g} \mathrm{~cm}^{-3}$ & 0.0 & -1.85 & -0.85 & -1.18 & -0.10 & -1.30 & +0.36 \\
\hline def $5 \times 10^{9} \mathrm{~g} \mathrm{~cm}^{-3}$ & 0.0 & -2.08 & -1.02 & -1.32 & +0.32 & -0.73 & +0.29 \\
\hline \multicolumn{8}{|c|}{$\operatorname{sub}($ L19) } \\
\hline $0.90 M_{\odot}, M_{\mathrm{He}}=0.15 M_{\odot}$ & 0.0 & -0.30 & +0.38 & +0.15 & +0.86 & -0.21 & +0.09 \\
\hline $0.95 M_{\odot}, M_{\mathrm{He}}=0.15 M_{\odot}$ & 0.0 & -0.88 & -0.08 & -0.30 & +0.61 & -0.41 & +0.05 \\
\hline $1.00 M_{\odot}, M_{\mathrm{He}}=0.10 \mathrm{M}_{\odot}$ & 0.0 & -0.88 & -0.09 & -0.33 & +0.53 & -0.39 & +0.03 \\
\hline $1.05 M_{\odot}, M_{\mathrm{He}}=0.10 \mathrm{M}_{\odot}$ & 0.0 & -1.19 & -0.32 & -0.54 & +0.41 & -0.41 & +0.06 \\
\hline $1.10 \mathrm{M}_{\odot}, M_{\mathrm{He}}=0.10 \mathrm{M}_{\odot}$ & -1.5 & -1.32 & -0.48 & -0.51 & +0.31 & -0.83 & -0.34 \\
\hline $1.15 M_{\odot}, M_{\mathrm{He}}=0.10 \mathrm{M}_{\odot}$ & 0.0 & -2.09 & -0.67 & -0.72 & +0.08 & -0.43 & +0.04 \\
\hline $1.20 M_{\odot}, M_{\mathrm{He}}=0.05 M_{\odot}$ & 0.0 & -1.93 & -0.70 & -0.79 & -0.12 & -0.52 & +0.12 \\
\hline \multicolumn{8}{|c|}{$\operatorname{sub}(\mathrm{S} 18)$} \\
\hline $0.85 M_{\odot}, \mathrm{C} / \mathrm{O}=50 / 50$ & -1.5 & -0.82 & +0.61 & +0.61 & +0.58 & -2.04 & -1.70 \\
\hline $0.90 M_{\odot}, \mathrm{C} / \mathrm{O}=50 / 50$ & -1.5 & -1.37 & +0.24 & +0.30 & +0.47 & -2.48 & -1.92 \\
\hline $1.00 M_{\odot}, \mathrm{C} / \mathrm{O}=50 / 50$ & -1.5 & -2.19 & -0.23 & -0.11 & +0.12 & -0.70 & -0.46 \\
\hline $1.10 \mathrm{M}_{\odot}, \mathrm{C} / \mathrm{O}=50 / 50$ & -1.5 & -3.01 & -0.63 & -0.44 & -0.17 & -0.55 & -0.30 \\
\hline $0.85 M_{\odot}, \mathrm{C} / \mathrm{O}=30 / 70$ & -1.5 & -0.46 & +0.75 & +0.79 & +0.64 & -1.51 & -1.55 \\
\hline $0.90 M_{\odot}, \mathrm{C} / \mathrm{O}=30 / 70$ & -1.5 & -1.17 & +0.30 & +0.42 & +0.52 & -2.30 & -1.89 \\
\hline $1.00 M_{\odot}, \mathrm{C} / \mathrm{O}=30 / 70$ & -1.5 & -2.14 & -0.20 & -0.02 & +0.19 & -0.80 & -0.54 \\
\hline $1.10 \mathrm{M}_{\odot}, \mathrm{C} / \mathrm{O}=30 / 70$ & -1.5 & -3.07 & -0.59 & -0.37 & -0.10 & -0.59 & -0.32 \\
\hline \multicolumn{8}{|c|}{$\operatorname{sub}(\mathrm{B} 19)$} \\
\hline $0.88 M_{\odot}, \xi_{\mathrm{CO}}=0.9$ & -1.5 & -0.52 & +0.42 & +0.66 & +0.48 & -2.77 & -1.85 \\
\hline $0.97 M_{\odot}, \xi_{\mathrm{CO}}=0.9$ & -1.5 & -1.29 & -0.09 & +0.25 & +0.30 & -1.55 & -1.59 \\
\hline $1.06 M_{\odot}, \xi_{\mathrm{CO}}=0.9$ & -1.5 & -1.94 & -0.46 & -0.07 & +0.02 & -0.82 & -0.70 \\
\hline $1.10 \mathrm{M}_{\odot}, \xi_{\mathrm{CO}}=0.9$ & -1.5 & -2.24 & -0.62 & -0.20 & -0.11 & -0.74 & -0.62 \\
\hline $1.15 M_{\odot}, \xi_{\mathrm{CO}}=0.9$ & -1.5 & -2.64 & -0.83 & -0.39 & -0.27 & -0.67 & -0.56 \\
\hline $0.88 M_{\odot}, \xi_{\mathrm{CO}}=0.0$ & -1.5 & -0.38 & +0.60 & +0.64 & +0.48 & -2.68 & -1.82 \\
\hline $0.97 M_{\odot}, \xi_{\mathrm{CO}}=0.0$ & -1.5 & -1.22 & +0.02 & +0.18 & +0.28 & -1.52 & -1.57 \\
\hline $1.06 M_{\odot}, \xi_{\mathrm{CO}}=0.0$ & -1.5 & -1.86 & -0.36 & -0.15 & -0.01 & -0.81 & -0.68 \\
\hline $1.10 \mathrm{M}_{\odot}, \xi_{\mathrm{CO}}=0.0$ & -1.5 & -2.16 & -0.53 & -0.28 & -0.13 & -0.75 & -0.61 \\
\hline $1.15 M_{\odot}, \xi_{\mathrm{CO}}=0.0$ & -1.5 & -2.56 & -0.75 & -0.46 & -0.29 & -0.73 & -0.60 \\
\hline
\end{tabular}


metallicity. The threshold metallicity imposed by simmering has variously been estimated to be $2 / 3 Z_{\odot}$ (Piro \& Bildsten 2008) or $1 / 3 Z_{\odot}$ (Martínez-Rodríguez et al. 2016). Both of these values are much larger than the most metal-rich stars in our sample. The $M_{\mathrm{Ch}}$ DDT models computed at sub-solar metallicity in Table 2 do not include simmering. Therefore, it is better to compare our observationally inferred yields to the solar-metallicity DDT models. From here on, we disregard the DDT models at sub-solar metallicity. The pure deflagration models are available only at solar metallicity, and the sub- $M_{\mathrm{Ch}}$ models are not subject to simmering.

Where possible, we show sub- $M_{\mathrm{Ch}}$ models interpolated to a metallicity of $Z=10^{-1.5} Z_{\odot}$ so that it is easier to compare with the observationally inferred yields (Section 4.2), which we tabulate at $[\mathrm{Fe} / \mathrm{H}]=-1.5$. However, some models are available only at solar metallicity. The second column in Table 3 specifies the metallicity at which the theoretical yields are given. Figure 6 represents solar-metallicity models in shades of red. Models interpolated to a metallicity of $Z=10^{-1.5} Z_{\odot}$ are shown in shades of blue.

The effect of metallicity is especially apparent in the $[\mathrm{Co} / \mathrm{Fe}]$ and $[\mathrm{Ni} / \mathrm{Fe}]$ sub- $M_{\mathrm{Ch}}$ yields of L19. The yields of the $1.10 \mathrm{M}_{\odot}$ model reflect a metallicity of $Z=10^{-1.5} Z_{\odot}$, but modeled WDs of higher and lower masses have solar metallicity. The solarmetallicity models show a smooth gradient in yields, but the low-metallicity model is offset to lower abundance ratios. This offset results from the neutron enhancement of highermetallicity models. The extra neutrons allow the creation of neutron-rich species, like ${ }^{58} \mathrm{Ni}$.

The choice of ignition parameters also influences the yields (see the discussion by Fisher \& Jumper 2015). In $M_{\mathrm{Ch}}$ models, a larger number of ignition sites translates to greater preexpansion of the WD. The resulting lower density leads to synthesis of more intermediate-mass elements, like $\mathrm{Si}$, and fewer Fe-group isotopes, such as ${ }^{56} \mathrm{Ni}$, which decays to ${ }^{56} \mathrm{Fe}$, and ${ }^{58} \mathrm{Ni}$, which is stable. Therefore, a greater number of ignition sites-especially off-center ignitions-leads to higher $[\mathrm{Si} / \mathrm{Fe}]$ yields and lower $[\mathrm{Ni} / \mathrm{Fe}]$ yields.

The most notable feature that distinguishes the yields of different classes of $\mathrm{SNe} \mathrm{Ia}$ is the difference in the $[\mathrm{Ni} / \mathrm{Fe}]$ ratio between near- $M_{\mathrm{Ch}}$ and sub- $M_{\mathrm{Ch}}$ explosions. Higher-mass WDs have higher densities, which permit more complete burning in nuclear statistical equilibrium, including electron captures that increase the neutron density. A high neutron density favors the creation of Fe-peak elements over intermediate-mass elements. Even within the sub- $M_{\mathrm{Ch}}$ simulations, WDs of higher mass (represented by darker shades in Figure 6) produce lower $[\mathrm{Si} / \mathrm{Fe}],[\mathrm{Ca} / \mathrm{Fe}]$, and $[\mathrm{Cr} / \mathrm{Fe}]$ ratios and higher $[\mathrm{Co} / \mathrm{Fe}]$ and $[\mathrm{Ni} / \mathrm{Fe}]$ ratios. The only simulations that predict $[\mathrm{Ni} / \mathrm{Fe}]<$ -0.01 are sub- $M_{\mathrm{Ch}}$ models. Furthermore, all of the metal-poor sub- $M_{\mathrm{Ch}}$ simulations predict $[\mathrm{Ni} / \mathrm{Fe}] \leqslant-0.30$.

\subsection{Comparison of Observationally Inferred Yields to Theoretical Yields}

The preceding discussion suggests that $[\mathrm{Ni} / \mathrm{Fe}]_{\mathrm{Ia}}$ is a potential indicator of whether $\mathrm{SNe}$ Ia result from the explosions of near- $M_{\mathrm{Ch}}$ or sub- $M_{\mathrm{Ch}}$ WDs. The observationally inferred yields for different $\mathrm{dSphs}$ range from $[\mathrm{Ni} / \mathrm{Fe}]_{\mathrm{Ia}}=-0.68_{-0.09}^{+0.07}$ (Ursa Minor) to $-0.13_{-0.04}^{+0.03}$ (Leo II). The inferred $[\mathrm{Ni} / \mathrm{Fe}]$ yields are best explained by sub- $M_{\mathrm{Ch}}$ detonations. The $[\mathrm{Ni} / \mathrm{Fe}]$ yields are not compatible with $M_{\mathrm{Ch}}$ DDTs or pure deflagarations. Under the assumption that Type Iax SNe are pure deflagrations, our results indicate that Type Iax $\mathrm{SNe}$ are not a major contributor to galactic chemical evolution. Other element ratios do not distinguish between near- $M_{\mathrm{Ch}}$ or sub- $M_{\mathrm{Ch}} \mathrm{SNe} \mathrm{Ia}$ as well as $[\mathrm{Ni} / \mathrm{Fe}]$. Nonetheless, the inferred yields for all elements and for all dSphs are compatible with sub- $M_{\mathrm{Ch}}$ explosions.

As discussed in Section 4.1, the WD mass influences the yield ratios. The observationally inferred $[\mathrm{Ni} / \mathrm{Fe}]_{\text {Ia }}$ yields best match some of the more massive sub- $M_{\mathrm{Ch}}$ WDs, specifically the S18 and L19 models with masses of $1.00-1.10 M_{\odot}$. McWilliam et al. (2018) pointed out that $[\mathrm{Si} / \mathrm{Fe}]$ is a strong indicator of WD mass. Our estimates of $[\mathrm{Si} / \mathrm{Fe}]_{\mathrm{Ia}}$ match the S18 and B19 models of WDs from $1.00 M_{\odot}$ to slightly larger than $1.15 M_{\odot}$. WDs of this mass are heavily outnumbered by lower-mass WDs in old populations. However, the inferred yields reflect the stellar population of the dSphs at the time of star formation, not the present time. With the exception of Leo II, the galaxies in this study formed the majority of their stars in 1-2 Gyr (Kirby et al. 2011; Weisz et al. 2014). The lifetime of a $1.7 M_{\odot}$ star is about $1 \mathrm{Gyr}$ (Padovani \& Matteucci 1993), and such a star would make a WD of $0.59 M_{\odot}$ (Kalirai et al. 2008). Therefore, there would be plenty of low-mass WDs during the active lifetime of the dSphs. However, the WDs must be given time to explode in order to relate this discussion to SNe Ia. Shen et al. (2017) modeled SNe Ia as prompt double detonations of WD binaries. In that scenario, $90 \%$ of SNe Ia arise from WDs of $>1 M_{\odot}$ in stellar populations with ages ranging from 0.3 to $1.0 \mathrm{Gyr}$. The percentage drops to $50 \%$ in stellar populations with ages in the range 1-3 Gyr, which is the time frame in which many dSphs formed most of their stars (Weisz et al. 2014).

The WD metallicity also has an effect on yield ratios (also discussed in Section 4.1). We inferred SN Ia yields as a function of metallicity. Figure 8 compares our inferences to the theoretical predictions. In general, $[\mathrm{Ni} / \mathrm{Fe}]$ is predicted to increase with $[\mathrm{Fe} / \mathrm{H}]$, but we inferred that the yield decreases with metallicity. However, the predicted metallicity dependence at $[\mathrm{Fe} / \mathrm{H}]<-1$ is very weak for WDs with masses greater than $1 M_{\odot}$. In fact, we might expect $[\mathrm{Ni} / \mathrm{Fe}]_{\mathrm{Ia}}$ to decrease with metallicity because the average mass of the exploding WDs decreases over time (Shen et al. 2017). As a result, $[\mathrm{Ni} / \mathrm{Fe}]_{\text {Ia }}$ could reflect the yields of lower-mass WDs as the galaxy ages and becomes more metal-rich. This hypothesis could be tested by convolving the exploding WD mass function expected for a given age with the predicted SN Ia yields. We save this analysis for the future.

S18 and B19 considered two variables that affected the SN Ia yields. S18 used two different C/O ratios, and B19 reduced the ${ }^{12} \mathrm{C}+{ }^{16} \mathrm{O}$ reaction rate by a factor of 10 . (S18 also experimented with the reaction rate, but we present only their results using the "standard" rate.) In most cases, these choices result in differences of Fe-peak abundance ratios on the order of $\lesssim 0.1$ dex. In fact, B19 specifically pointed out that abundance ratios are less sensitive than absolute abundances to the reaction rate. Therefore, the $\mathrm{C} / \mathrm{O}$ ratio and the $\mathrm{C}+\mathrm{O}$ reaction rate do not affect our conclusion about the dominant role of sub- $M_{\mathrm{Ch}}$ SNe Ia in dSphs.

Our conclusions are valid only for SNe Ia that occurred during the time in which the dSphs were forming stars. The dSphs in our sample formed the middle two thirds of their stars in durations ranging from $1 \mathrm{Gyr}$ in Sculptor to $5 \mathrm{Gyr}$ in Leo II (Weisz et al. 2014). Some population synthesis models predict that single-degenerate, $M_{\mathrm{Ch}}$ SNe Ia can be delayed by 


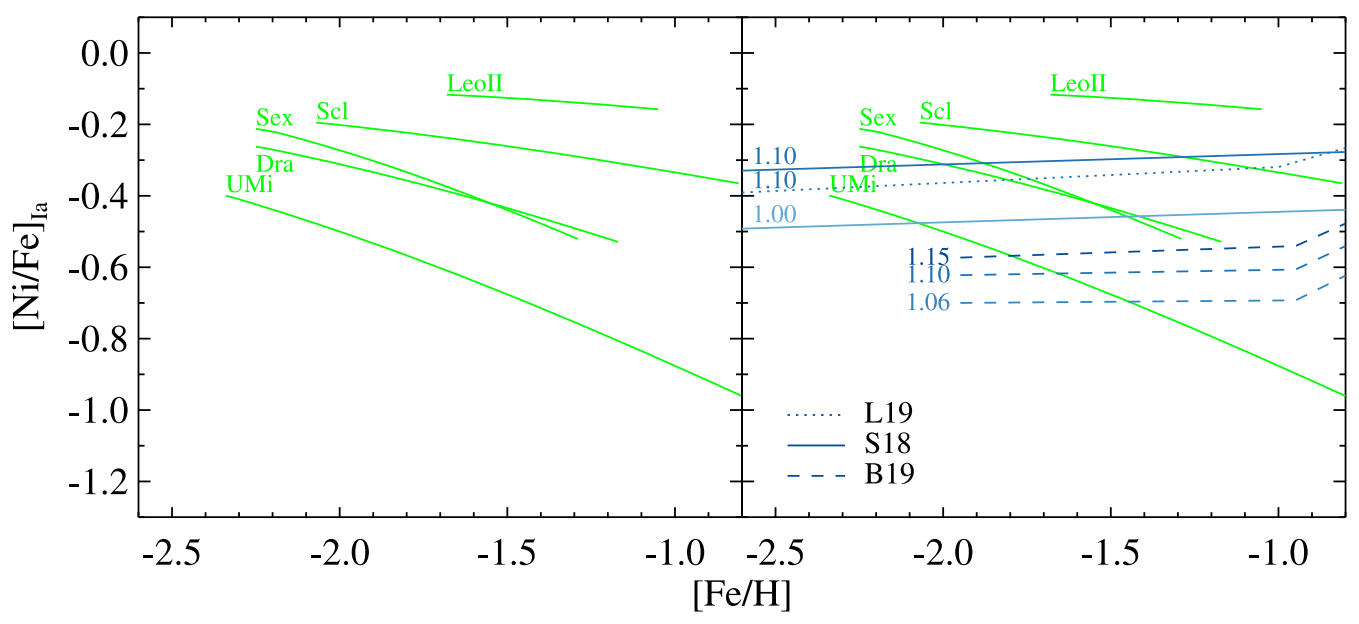

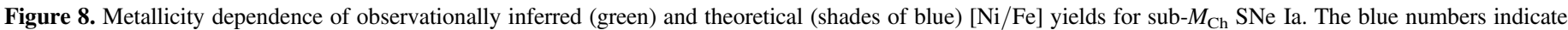

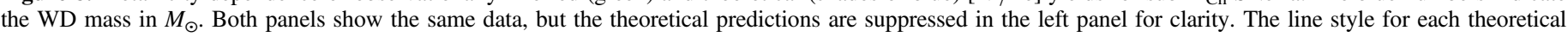
prediction reflects the study from which it came, and the shading indicates the initial WD mass, consistent with Figure 6.

0.6-1.9 Gyr (Ruiter et al. 2009, 2011; Yungelson 2010). However, all of these long-delayed models fall short of explaining the cosmological rate of $\mathrm{SNe}$ Ia by at least two orders of magnitudes (Nelemans et al. 2013). Therefore, our measurements are likely sensitive to the "standard" models of $\mathrm{SNe}$ Ia that have shorter delays (i.e., those reviewed by Maoz et al. 2014) . Even those models fall short of the observed rate of SNe Ia, but the discrepancy is less than for the long-delayed models. Regardless, it is important to note that our measurements are not sensitive to SNe Ia that are delayed by more than the peak of star formation in the dSphs. One example of such a model is one that takes into account the effects of low metallicity on the accretion rate and winds from accreting WDs (Kobayashi \& Nomoto 2009). We discuss this model further at the end of Section 5.

\section{Discussion}

We have used a galactic archaeological approach to inferring the explosion mechanism of SNe Ia. We concluded that the abundances - specifically $[\mathrm{Ni} / \mathrm{Fe}]$ - in ancient dSphs are most consistent with sub- $M_{\mathrm{Ch}}$ explosions. Our approach assumed that the galaxies were well-mixed at all times and that the IMF did not change. We made no assumptions about the form of the IMF. We also did not presuppose any theoretical SN yield for any abundance ratio except for $[\mathrm{Mg} / \mathrm{Fe}]$.

Ours is not the first galactic archaeological study to address the nature of SNe Ia. Most of the previous studies focused on Mn because it is particularly sensitive to the mass and metallicity of the exploding WD (Seitenzahl et al. 2013a). In fact, we will present our own Mn abundance measurements in the near future. North et al. (2012) measured Mn abundances in four dSphs. They measured sub-solar abundances of $[\mathrm{Mn} / \mathrm{Fe}]$ in all four dSphs, and they also found a trend with $[\mathrm{Fe} / \mathrm{H}]$. They concluded that metallicity-dependent yields are the best explanation for their observations. However, they did not have the benefit of the sizable menu of theoretical yields available today. As a result, they did not consider the mass of the exploding WD.

Perets et al. (2010) discovered a new kind of explosion they called "Ca-rich SNe" because of their high $\mathrm{Ca} / \mathrm{O}$ nebular line ratios. Their existence in the luminosity gap between novae and SNe led Kasliwal et al. (2012) to call them "Ca-rich gap transients." Our observations show that $[\mathrm{Ca} / \mathrm{Fe}]$ declines more slowly than $[\mathrm{Si} / \mathrm{Fe}]$ in dwarf galaxies. As a result, we also find that $[\mathrm{Ca} / \mathrm{Fe}]_{\mathrm{Ia}}$ is significantly higher than $[\mathrm{Si} / \mathrm{Fe}]_{\mathrm{Ia}}$. One interpretation is that $\mathrm{SNe}$ Ia produce a higher ratio of $[\mathrm{Ca} / \mathrm{Fe}]$ than $[\mathrm{Si} / \mathrm{Fe}]$. In fact, this is the behavior predicted by the sub- $M_{\mathrm{Ch}}$ models of $\mathrm{S} 18$ and $\mathrm{B} 19$. An alternative explanation is that there is a second class of delayed explosions, i.e., Ca-rich transients. While our results do not require an additional nucleosynthetic source beyond $\mathrm{CCSNe}$ and $\mathrm{SNe} \mathrm{Ia}$, we cannot rule out that $\mathrm{Ca}$-rich gap transients contribute to the chemical evolution of dSphs.

So far, we have discounted Type Iax SNe. Kobayashi et al. (2015) and Cescutti \& Kobayashi (2017) both explored the effect of Type Iax SNe on the chemical evolution of dSphs. They used measurements of $\alpha$ and Fe-peak elements from North et al. (2012), Venn et al. (2012), Ural et al. (2015), and others. With the benefit of a one-zone chemical evolution model, Kobayashi et al. concluded that a mixture of Type Iax and sub- $M_{\mathrm{Ch}}$ explosions best explains the evolution of $[\mathrm{Mn} / \mathrm{Fe}]$. Cescutti \& Kobayashi abandoned the assumption of well-mixed gas. Instead, they introduced stochasticity into their models as a way to approximate the spread of $[\mathrm{Mn} / \mathrm{Fe}]$ at a given $[\mathrm{Fe} / \mathrm{H}]$. They interpreted the observations of Ursa Minor as having a spread consistent with their model, which invokes multiple sub-classes of SN Ia.

On the other hand, McWilliam et al. (2018) presented a different interpretation of the evolution of Fe-peak elements, including $[\mathrm{Ni} / \mathrm{Fe}]$, in Ursa Minor at fixed $[\mathrm{Fe} / \mathrm{H}]$. They relied on Cohen \& Huang's (2010) high-resolution spectroscopic measurements of 10 stars. In contrast to Cescutti \& Kobayashi (2017), they did not find evidence for a spread in $[\mathrm{Mn} / \mathrm{Fe}]$. However, COS 171, the star with the highest metallicity in the sample, has very low $[\mathrm{Mn} / \mathrm{Fe}]$ and $[\mathrm{Ni} / \mathrm{Fe}]$ ratios. The unusual abundances of this star are reminiscent of Car 612 in the Carina dSph (Venn et al. 2012). McWilliam et al. concluded that COS 171 was the result of the explosion of a $0.95 M_{\odot}$ WD. This interpretation for the abundances of one star matches our interpretation for the abundances of the ensemble of stars in several dSphs. In other words, COS 171 is not the result of a rare type of SN Ia. Instead, sub- $M_{\mathrm{Ch}}$ explosions are typical in dSphs.

Our conclusion is somewhat compatible with abundances of cold gas at high redshift. Lu et al. (1996) and Cooke et al. (2015) 


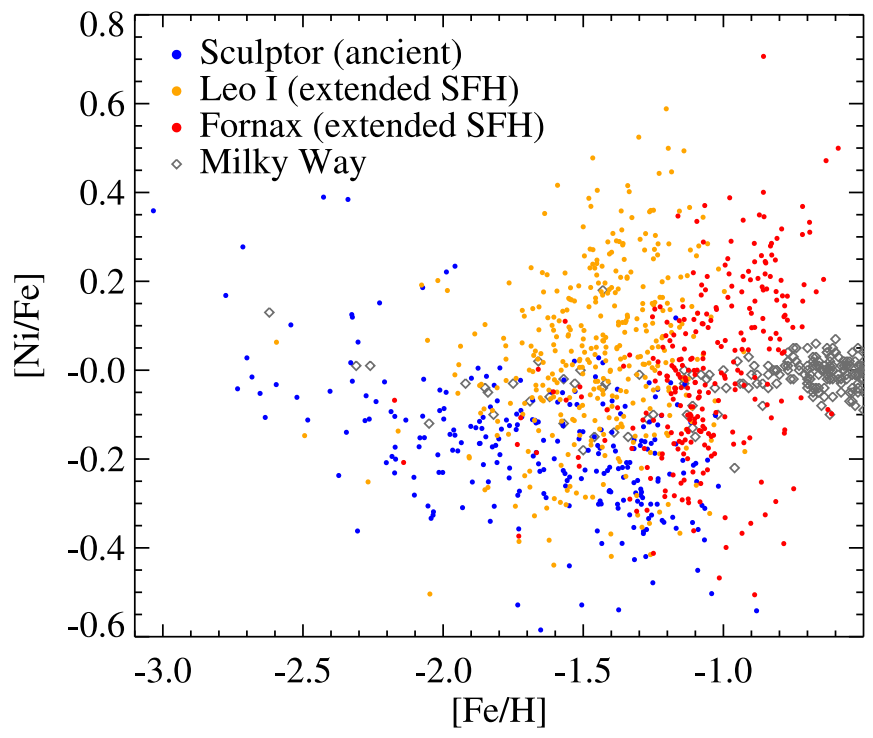

Figure 9. Evolution of $[\mathrm{Ni} / \mathrm{Fe}]$ with $[\mathrm{Fe} / \mathrm{H}]$ in three dSphs. Only stars with uncertainties less than 0.2 dex are shown. The $[\mathrm{Ni} / \mathrm{Fe}]$ evolution in the Milky Way (Bensby et al. 2014) is shown in gray diamonds.

measured $\mathrm{Ni}$ abundances in damped Ly $\alpha$ (DLA) systems to be in the range $-0.4 \lesssim[\mathrm{Ni} / \mathrm{Fe}] \lesssim-0.1 .^{10}$ The DLAs studied were all metal-poor $(-2.4 \leqslant[\mathrm{Fe} / \mathrm{H}] \leqslant-0.8)$. The agreement is notable because we used a galactic archaeological technique at $z=0$, but the DLA study was conducted at redshifts as high as $z=3$. These abundances suggest that nucleosynthesis and chemical evolution are similar across small, metal-poor galaxies.

Our findings regarding dwarf galaxies may not be extensible to more massive galaxies, like the MW. The $[\mathrm{Ni} / \mathrm{Fe}]$ trend in the MW is somewhat complex (e.g., Venn et al. 2004; Bensby et al. 2014; Hasselquist et al. 2017; Lomaeva et al. 2019). In the low-metallicity $([\mathrm{Fe} / \mathrm{H}] \lesssim-1)$ halo, the average value of $[\mathrm{Ni} / \mathrm{Fe}]$ is about -0.1 . At $[\mathrm{Fe} / \mathrm{H}]>-1$, the dispersion in $[\mathrm{Ni} / \mathrm{Fe}]$ suddenly decreases, and the mean value is 0.0 until $[\mathrm{Fe} / \mathrm{H}] \approx 0.0$. (See Figure 9.) At super-solar metallicities, $[\mathrm{Ni} / \mathrm{Fe}]$ smoothly increases with $[\mathrm{Fe} / \mathrm{H}]$. One interpretation of this behavior is that the MW halo is composed of dSphs with chemical evolution similar to Sagittarius (e.g., Hasselquist et al. 2017) and those studied in this work. As a result, their nucleosynthesis matches our interpretation, including the dominance of sub- $M_{\mathrm{Ch}}$ SNe Ia. However, the MW disk has a different type of stellar population. In fact, Seitenzahl et al. (2013a) previously argued that $[\mathrm{Mn} / \mathrm{Fe}]$ abundances in the MW disk are too high to be explained by sub- $M_{\mathrm{Ch}} \mathrm{SNe}$ Ia. Perhaps the SNe Ia that exploded in the MW disk were of a different class than those that exploded in the halo.

Conroy et al. (2014) found that giant elliptical galaxies with lower velocity dispersion (i.e., those with lower $[\alpha / \mathrm{Fe}]$ ) have lower integrated-light abundances of $[\mathrm{Ni} / \mathrm{Fe}]$. This finding corroborates the idea that galaxies with more SNe Ia have lower $[\mathrm{Ni} / \mathrm{Fe}]$ ratios. One interpretation of this result is that SNe Ia are exploding below $M_{\mathrm{Ch}}$ even in giant elliptical

\footnotetext{
${ }^{10}$ However, Prochaska \& Wolfe (2002) measured $-0.2 \lesssim[\mathrm{Ni} / \mathrm{Fe}] \lesssim+0.2$. The Ni absorption lines measured in DLAs are very weak, which leads to large uncertainties in the $[\mathrm{Ni} / \mathrm{Fe}]$ ratios. Furthermore, both $\mathrm{Lu}$ et al. (1996) and Prochaska \& Wolfe (2002) mentioned that the oscillator strengths of the $\mathrm{Ni}$ transitions are not well known.
}

galaxies. However, the $[\mathrm{Ni} / \mathrm{Fe}]$ ratios found in those galaxies are much larger than those found in this work for dwarf galaxies. Furthermore, Conroy et al. found a weaker decrease in $[\mathrm{Mn} / \mathrm{Fe}]$ with decreasing velocity dispersion. Therefore, the interpretation of the integrated-light Fe-peak abundances for giant galaxies is not as straightforward as for the dwarf galaxies.

At even higher mass scales, Fe-peak abundances may be measured in the intracluster gas in galaxy clusters with X-ray spectroscopy. Mernier et al. (2016a, 2016b) measured the abundances of elements including $\mathrm{Mn}, \mathrm{Fe}$, and $\mathrm{Ni}$ in dozens of galaxy clusters. They concluded that the super-solar values of $[\mathrm{Mn} / \mathrm{Fe}]$ and $[\mathrm{Ni} / \mathrm{Fe}]$ precluded a significant contribution of sub- $M_{\mathrm{Ch}}$ SNe Ia. Likewise, the Hitomi Collaboration (2017) measured the abundances of Fe-peak elements from X-ray spectra of the hot intracluster gas in the Perseus galaxy cluster. They found solar ratios of $[\mathrm{Mn} / \mathrm{Fe}]$ and $[\mathrm{Ni} / \mathrm{Fe}]$. Similar to Mernier et al. (2016b), they concluded that the dominant source of Fe-peak elements in the cluster is either near- $M_{\mathrm{Ch}} \mathrm{SNe}$ Ia or a roughly equal mixture of near- $M_{\mathrm{Ch}}$ and sub- $M_{\mathrm{Ch}} \mathrm{SNe}$ Ia. Bulk outflows from galaxies enriched the hot intracluster gas whereas stars formed from the cold interstellar gas that did not escape the galaxy. Therefore, it may not be advisable to compare galaxy cluster gas abundances to stellar abundances. Regardless, the X-ray measurements from galaxy clusters potentially corroborate a scenario in which the dominant mode of SNe Ia transitions from sub- $M_{\mathrm{Ch}}$ at low galaxy mass (or low metallicity) to near- $M_{\mathrm{Ch}}$ at higher galaxy mass.

Kobayashi et al. $(1998,2015)$ and Kobayashi \& Nomoto (2009) predicted that the rates and explosion mechanisms of $\mathrm{SNe}$ Ia depend on metallicity. Specifically, winds from WDs are more intense at higher metallicity. Winds allow the mass accretion rate to proceed slowly enough to be stable (Hachisu et al. 1996). Therefore, metallicities of $[\mathrm{Fe} / \mathrm{H}] \gtrsim-1.1$ could permit a single-degenerate WD to grow to $M_{\mathrm{Ch}}$ via mass accretion from a red giant companion. It is possible that the dominant SN Ia mechanism transitions from sub- $M_{\mathrm{Ch}}$ (or even Type Iax, as suggested by Kobayashi et al. 2015) at low metallicities to near- $M_{\mathrm{Ch}}$ at high metallicities. The observations of Fe-peak abundances in some dwarf galaxies and in large galaxies, like the MW, are consistent with this paradigm.

An alternative hypothesis is that the dominant class of SN Ia depends not on metallicity but on SFH. Figure 9 shows the $[\mathrm{Ni} / \mathrm{Fe}]$ evolution in Sculptor, Leo I, and Fornax. We have not included Leo I or Fornax in our discussion until now because they do not conform well to our simple bi-linear model of chemical evolution (Section 3.1). The average metallicities of Leo I and Fornax are higher than that of Sculptor, but their metallicity ranges overlap. It is very interesting that Leo I has higher values of $[\mathrm{Ni} / \mathrm{Fe}]$ than Sculptor at the same metallicity. It seems that metallicity is not the only variable that controls $[\mathrm{Ni} / \mathrm{Fe}]$. Even more importantly, abundance ratios above $[\mathrm{Ni} / \mathrm{Fe}] \sim+0.1$ cannot be explained by any of the sub- $M_{\mathrm{Ch}}$ models listed in Table 3.

Weisz et al. (2014) measured the SFH of Sculptor to be exclusively ancient. The SFHs of Leo I and Fornax are extended. Both formed stars steadily for nearly the entire age of the universe. Even so, both galaxies show decreasing $[\alpha / \mathrm{Fe}]$ with increasing $[\mathrm{Fe} / \mathrm{H}]$ (Letarte et al. 2010; Kirby et al. 2011; Lemasle et al. 2014), which is evidence for increasing enrichment by SNe Ia. Therefore, the enhanced, rising trends of $[\mathrm{Ni} / \mathrm{Fe}]$ for Leo I and Fornax cannot be explained by a high 
ratio of CCSNe to SNe Ia. Instead, it could be that a different, more delayed class of SN Ia dominates the $[\mathrm{Ni} / \mathrm{Fe}]$ evolution in Leo I and Fornax, whereas the SNe Ia in Sculptor are more prompt. Kobayashi \& Nomoto (2009) presented a scenario where single-degenerate, $M_{\mathrm{Ch}} \mathrm{SNe}$ Ia (i.e., those that produce $[\mathrm{Ni} / \mathrm{Fe}]>0$ ) explode later than double-degenerate $\mathrm{SNe} \mathrm{Ia}$ (i.e., those that produce $[\mathrm{Ni} / \mathrm{Fe}]<0$ ). It is possible that the $[\mathrm{Ni} / \mathrm{Fe}]$ evolution in Leo I and Fornax is evidence of a transition from double-degenerate, sub- $M_{\mathrm{Ch}}$ to singledegenerate, near- $M_{\mathrm{Ch}} \mathrm{SNe}$ Ia.

\section{Summary}

We have used our previously published catalogs of $\alpha$ and Fe-peak abundances in dSphs (Kirby et al. 2010, 2018) to infer properties of SNe Ia in dwarf galaxies. We used the evolution of $[\mathrm{Mg} / \mathrm{Fe}]$ to quantify the fraction $\left(f_{\mathrm{Ia}}\right)$ of $\mathrm{Fe}$ produced in $\mathrm{SNe}$ Ia. We applied this ratio to the observed evolution of other element ratios, including $[\mathrm{Ni} / \mathrm{Fe}]$, to infer the yields of $\mathrm{SNe} \mathrm{Ia}$, e.g., $[\mathrm{Ni} / \mathrm{Fe}]_{\mathrm{Ia}}$. In the dSphs with short SFHs, the observationally inferred yields of $[\mathrm{Si} / \mathrm{Fe}]_{\mathrm{Ia}},[\mathrm{Ca} / \mathrm{Fe}]_{\mathrm{Ia}},[\mathrm{Co} / \mathrm{Fe}]_{\mathrm{Ia}}$, and $[\mathrm{Ni} / \mathrm{Fe}]_{\mathrm{Ia}}$ are sub-solar, whereas $[\mathrm{Cr} / \mathrm{Fe}]_{\mathrm{Ia}}$ is approximately solar. These yields are consistent with the detonations of $\sim 1.0 M_{\odot}$ WDs. However, the $[\mathrm{Ni} / \mathrm{Fe}]_{\mathrm{Ia}}$ yields are inconsistent with the explosions of near- $M_{\mathrm{Ch}}$ WDs. Two dSphs with more extended SFHs (Fornax and Leo I) contain stars with enhanced $[\mathrm{Ni} / \mathrm{Fe}]$ that cannot be explained by sub- $M_{\mathrm{Ch}} \mathrm{SNe}$ Ia.

We concluded that the dominant explosion mechanism of SNe Ia that occurred before the end of star formation in shortlived dwarf galaxies is the detonation of a sub- $M_{\mathrm{Ch}}$ WD. This conclusion cannot necessarily be generalized to the total population of SNe Ia. Specifically, the evolution of $[\mathrm{Ni} / \mathrm{Fe}]$ in the MW disk and in dSphs with extended SFHs is different from that in ancient dSphs.

We did not observe a strong evolution of SN Ia yields with metallicity, but theoretical models do not predict a strong metallicity dependence at $[\mathrm{Fe} / \mathrm{H}]<-1$, which includes the vast majority of stars in our sample. The weak metallicity dependence we did observe might instead confirm the prediction that lower-mass, sub- $M_{\mathrm{Ch}}$ WDs explode at later times (Shen et al. 2017).

We also observed a marked difference in the $[\mathrm{Ni} / \mathrm{Fe}]$ evolution between galaxies with different SFHs. Specifically, $[\mathrm{Ni} / \mathrm{Fe}]$ is sub-solar in ancient galaxies, but $[\mathrm{Ni} / \mathrm{Fe}]$ is higher and increases with $[\mathrm{Fe} / \mathrm{H}]$ in galaxies that formed stars for several Gyr. The differences could reflect a shift in the dominant type of $\mathrm{SN}$ Ia (double-degenerate to singledegenerate and sub- $M_{\mathrm{Ch}}$ to $M_{\mathrm{Ch}}$ ) for galaxies with more extended SFHs.

In the future, we will measure $\mathrm{Mn}$, which is perhaps the element most sensitive to the mass and metallicity of the exploding WD. The evolution of $[\mathrm{Mn} / \mathrm{Fe}]$ will be a powerful check on our conclusions, which were based mostly on $[\mathrm{Ni} / \mathrm{Fe}]$. Our data set also has several other possible uses, such as constructing models of chemical evolution that account for a range of WD masses (see Section 4.2). The data might also be useful in deducing the SN Ia delay time distribution.

We thank Marten van Kerkwijk, Julianne Dalcanton, Ivo Seitenzahl, Robert Fisher, Ken'ichi Nomoto, Mansi Kasliwal, Alex Ji, and Norbert Werner for insightful conversations. We also thank the anonymous referee for a helpful report. This material is based upon work supported by the National Science
Foundation under grant No. AST-1614081. E.N.K. gratefully acknowledges support from a Cottrell Scholar award administered by the Research Corporation for Science Advancement as well as funding from generous donors to the California Institute of Technology.

We are grateful to the many people who have worked to make the Keck Telescope and its instruments a reality and to operate and maintain the Keck Observatory. The authors wish to extend special thanks to those of Hawaiian ancestry on whose sacred mountain we are privileged to be guests. Without their generous hospitality, none of the observations presented herein would have been possible. We express our deep gratitude to the staff at academic and telescope facilities whose labor maintains spaces for scientific inquiry.

Facility: Keck:II (DEIMOS).

Software: MOOG (Sneden 1973; Sneden et al. 2012).

\section{ORCID iDs}

Evan N. Kirby (1) https://orcid.org/0000-0001-6196-5162 Maria Bergemann (iD https://orcid.org/0000-0002-9908-5571

Ken J. Shen (iD https://orcid.org/0000-0002-9632-6106 Anthony L. Piro (i) https://orcid.org/0000-0001-6806-0673 Andrew McWilliam (iD https://orcid.org/0000-00020765-431X

\section{References}

Arnett, W. D. 1969, Ap\&SS, 5, 180

Asplund, M., Grevesse, N., Sauval, A. J., \& Scott, P. 2009, ARA\&A, 47, 481 Badenes, C., Borkowski, K. J., Hughes, J. P., Hwang, U., \& Bravo, E. 2006, ApJ, 645, 1373

Bensby, T., Feltzing, S., \& Oey, M. S. 2014, A\&A, 562, A71

Bergemann, M., \& Cescutti, G. 2010, A\&A, 522, A9

Bergemann, M., Pickering, J. C., \& Gehren, T. 2010, MNRAS, 401, 1334

Blondin, S., Dessart, L., \& Hillier, D. J. 2018, MNRAS, 474, 3931

Bloom, J. S., Kasen, D., Shen, K. J., et al. 2012, ApJL, 744, L17

Borkowski, K. J., Reynolds, S. P., Green, D. A., et al. 2010, ApJL, 724, L161

Borkowski, K. J., Reynolds, S. P., Hwang, U., et al. 2013, ApJL, 771, L9

Bravo, E., Badenes, C., \& Martínez-Rodríguez, H. 2019, MNRAS, 482, 4346, (B19)

Brooks, J., Bildsten, L., Schwab, J., \& Paxton, B. 2016, ApJ, 821, 28

Byrohl, C., Fisher, R. T., \& Townsley, D. M. 2019, ApJ, 878, 67

Cao, Y., Kulkarni, S. R., Howell, D. A., et al. 2015, Natur, 521, 328

Cescutti, G., \& Kobayashi, C. 2017, A\&A, 607, A23

Chamulak, D. A., Brown, E. F., Timmes, F. X., \& Dupczak, K. 2008, ApJ, 677,160

Cohen, J. G., \& Huang, W. 2010, ApJ, 719, 931

Conroy, C., Graves, G. J., \& van Dokkum, P. G. 2014, ApJ, 780, 33

Cooke, R. J., Pettini, M., \& Jorgenson, R. A. 2015, ApJ, 800, 12

Dave, P., Kashyap, R., Fisher, R., et al. 2017, ApJ, 841, 58

De, K., Kasliwal, M. M., Polin, A., et al. 2019, ApJL, 873, L18

Escala, I., Wetzel, A., Kirby, E. N., et al. 2018, MNRAS, 474, 2194

Faber, S. M., Phillips, A. C., Kibrick, R. I., et al. 2003, in Proc. SPIE, 841 ed. M. Iye \& A. F. M. Moorwood, 1657

Fink, M., Kromer, M., Seitenzahl, I. R., et al. 2014, MNRAS, 438, 1762

Fisher, R., \& Jumper, K. 2015, ApJ, 805, 150

Foley, R. J., Challis, P. J., Chornock, R., et al. 2013, ApJ, 767, 57

Gaia Collaboration, Brown, A. G. A., Vallenari, A., et al. 2018, A\&A, 616, A1 Ganeshalingam, M., Li, W., Filippenko, A. V., et al. 2012, ApJ, 751, 142

Giammichele, N., Bergeron, P., \& Dufour, P. 2012, ApJS, 199, 29

Gilfanov, M., \& Bogdán, Á 2010, Natur, 463, 924

Gilmore, G., \& Wyse, R. F. G. 1991, ApJL, 367, L55

Goldstein, D. A., \& Kasen, D. 2018, ApJL, 852, L33

Grefenstette, B. W., Fryer, C. L., Harrison, F. A., et al. 2017, ApJ, 834, 19

Guillochon, J., Dan, M., Ramirez-Ruiz, E., \& Rosswog, S. 2010, ApJL, 709, L64

Hachinger, S., Mazzali, P. A., Sullivan, M., et al. 2013, MNRAS, 429, 2228

Hachisu, I., Kato, M., \& Nomoto, K. 1996, ApJL, 470, L97

Hamuy, M., Phillips, M. M., Suntzeff, N. B., et al. 2003, Natur, 424, 651

Hasselquist, S., Shetrone, M., Smith, V., et al. 2017, ApJ, 845, 162 
Heger, A., \& Woosley, S. E. 2010, ApJ, 724, 341

Hillebrandt, W., Kromer, M., Röpke, F. K., \& Ruiter, A. J. 2013, FrPhy, 8, 116

Hitomi Collaboration 2017, Natur, 551, 478

Hogg, D. W., Bovy, J., \& Lang, D. 2010, arXiv:1008.4686

Hosseinzadeh, G., Sand, D. J., Valenti, S., et al. 2017, ApJL, 845, L11

Iwamoto, K., Brachwitz, F., Nomoto, K., et al. 1999, ApJS, 125, 439

Kalirai, J. S., Hansen, B. M. S., Kelson, D. D., et al. 2008, ApJ, 676, 594

Kasliwal, M. M., Kulkarni, S. R., Gal-Yam, A., et al. 2012, ApJ, 755, 161

Katz, M. P., \& Zingale, M. 2019, ApJ, 874, 169

Khokhlov, A. M. 1991, A\&A, 245, 114

Kirby, E. N., Cohen, J. G., Smith, G. H., et al. 2011, ApJ, 727, 79

Kirby, E. N., Guhathakurta, P., Simon, J. D., et al. 2010, ApJS, 191, 352

Kirby, E. N., Xie, J. L., Guo, R., Kovalev, M., \& Bergemann, M. 2018, ApJS, 237, 18

Kleinman, S. J., Kepler, S. O., Koester, D., et al. 2013, ApJS, 204, 5

Kobayashi, C., \& Nomoto, K. 2009, ApJ, 707, 1466

Kobayashi, C., Nomoto, K., \& Hachisu, I. 2015, ApJL, 804, L24

Kobayashi, C., Tsujimoto, T., Nomoto, K., Hachisu, I., \& Kato, M. 1998, ApJL, 503, L155

Kollmeier, J. A., Chen, P., Dong, S., et al. 2019, MNRAS, 486, 3041

Kromer, M., Ohlmann, S. T., Pakmor, R., et al. 2015, MNRAS, 450, 3045

Lemasle, B., de Boer, T. J. L., Hill, V., et al. 2014, A\&A, 572, A88

Letarte, B., Hill, V., Tolstoy, E., et al. 2010, A\&A, 523, A17

Leung, S.-C., \& Nomoto, K. 2018, ApJ, 861, 143

Leung, S.-C., \& Nomoto, K. 2019, ApJS, submitted (arXiv:1901.10007)

Li, W., Bloom, J. S., Podsiadlowski, P., et al. 2011, Natur, 480, 348

Lomaeva, M., Jönsson, H., Ryde, N., Schultheis, M., \& Thorsbro, B. 2019, A\&A, 625, A141

Lopez, L. A., Grefenstette, B. W., Reynolds, S. P., et al. 2015, ApJ, 814, 132

Lu, L., Sargent, W. L. W., Barlow, T. A., Churchill, C. W., \& Vogt, S. S. 1996, ApJS, 107, 475

Maeda, K., Röpke, F. K., Fink, M., et al. 2010, ApJ, 712, 624

Maguire, K. 2017, in Handbook of Supernovae, ed. A. Alsabti \& P. Murdin (Berlin: Springer), 293

Maguire, K., Taubenberger, S., Sullivan, M., \& Mazzali, P. A. 2016, MNRAS, 457, 3254

Mannucci, F., Della Valle, M., \& Panagia, N. 2006, MNRAS, 370, 773

Maoz, D., \& Graur, O. 2017, ApJ, 848, 25

Maoz, D., Mannucci, F., \& Brandt, T. D. 2012, MNRAS, 426, 3282

Maoz, D., Mannucci, F., \& Nelemans, G. 2014, ARA\&A, 52, 107

Marion, G. H., Brown, P. J., Vinkó, J., et al. 2016, ApJ, 820, 92

Martínez-Rodríguez, H., Badenes, C., Yamaguchi, H., et al. 2017, ApJ, 843, 35

Martínez-Rodríguez, H., Piro, A. L., Schwab, J., \& Badenes, C. 2016, ApJ, 825,57

McWilliam, A., Piro, A. L., Badenes, C., \& Bravo, E. 2018, ApJ, 857, 97

Mernier, F., de Plaa, J., Pinto, C., et al. 2016a, A\&A, 592, A157

Mernier, F., de Plaa, J., Pinto, C., et al. 2016b, A\&A, 595, A126

Nelemans, G., Toonen, S., \& Bours, M. 2013, in IAU Symp. 281, Binary Paths to Type Ia Supernovae Explosions, ed. R. Di Stefano, M. Orio, \& M. Moe (Cambridge: Cambridge Univ. Press), 225

Nomoto, K. 1982, ApJ, 257, 780

Nomoto, K., Thielemann, F. K., \& Wheeler, J. C. 1984a, ApJL, 279, L23

Nomoto, K., Thielemann, F. K., \& Yokoi, K. 1984b, ApJ, 286, 644

Nomoto, K., Tominaga, N., Umeda, H., Kobayashi, C., \& Maeda, K. 2006, NuPhA, 777, 424

North, P., Cescutti, G., Jablonka, P., et al. 2012, A\&A, 541, A45

Padovani, P., \& Matteucci, F. 1993, ApJ, 416, 26

Pakmor, R., Kromer, M., Taubenberger, S., et al. 2012, ApJL, 747, L10

Pakmor, R., Kromer, M., Taubenberger, S., \& Springel, V. 2013, ApJL, 770, L8

Perets, H. B., Gal-Yam, A., Mazzali, P. A., et al. 2010, Natur, 465, 322

Perlmutter, S., Aldering, G., Goldhaber, G., et al. 1999, ApJ, 517, 565
Phillips, M. M. 1993, ApJL, 413, L105

Piersanti, L., Bravo, E., Cristallo, S., et al. 2017, ApJL, 836, L9

Piro, A. L., \& Bildsten, L. 2008, ApJ, 673, 1009

Pompéia, L., Hill, V., Spite, M., et al. 2008, A\&A, 480, 379

Post, S., Park, S., Badenes, C., et al. 2014, ApJL, 792, L20

Prochaska, J. X., \& Wolfe, A. M. 2002, ApJ, 566, 68

Raddi, R., Hollands, M. A., Gänsicke, B. T., et al. 2018a, MNRAS, 479, L96

Raddi, R., Hollands, M. A., Koester, D., et al. 2018b, ApJ, 858, 3

Raddi, R., Hollands, M. A., Koester, D., et al. 2019, MNRAS, in press

Riess, A. G., Filippenko, A. V., Challis, P., et al. 1998, AJ, 116, 1009

Röpke, F. K., Kromer, M., Seitenzahl, I. R., et al. 2012, ApJL, 750, L19

Ruiter, A. J., Belczynski, K., \& Fryer, C. 2009, ApJ, 699, 2026

Ruiter, A. J., Belczynski, K., Sim, S. A., et al. 2011, MNRAS, 417, 408

Saio, H., \& Nomoto, K. 1985, A\&A, 150, L21

Saio, H., \& Nomoto, K. 2004, ApJ, 615, 444

Salpeter, E. E. 1955, ApJ, 121, 161

Sbordone, L., Bonifacio, P., Buonanno, R., et al. 2007, A\&A, 465, 815

Scalzo, R. A., Ruiter, A. J., \& Sim, S. A. 2014, MNRAS, 445, 2535

Schaefer, B. E., \& Pagnotta, A. 2012, Natur, 481, 164

Seitenzahl, I. R., Cescutti, G., Röpke, F. K., Ruiter, A. J., \& Pakmor, R. 2013a, A\&A, 559, L5

Seitenzahl, I. R., Ciaraldi-Schoolmann, F., Röpke, F. K., et al. 2013b, MNRAS, 429, 1156

Seitenzahl, I. R., \& Townsley, D. M. 2017, Handbook of Supernovae, ed. A. Alsabti \& P. Murdin, (Berlin: Springer), arXiv:1704.00415

Shappee, B. J., Piro, A. L., Stanek, K. Z., et al. 2018, ApJ, 855, 6

Shen, K. J., \& Bildsten, L. 2007, ApJ, 660, 1444

Shen, K. J., Bildsten, L., Kasen, D., \& Quataert, E. 2012, ApJ, 748, 35

Shen, K. J., Boubert, D., Gänsicke, B. T., et al. 2018a, ApJ, 865, 15

Shen, K. J., Kasen, D., Miles, B. J., \& Townsley, D. M. 2018b, ApJ, 854, 52

Shen, K. J., \& Moore, K. 2014, ApJ, 797, 46

Shen, K. J., Toonen, S., \& Graur, O. 2017, ApJL, 851, L50

Shetrone, M., Venn, K. A., Tolstoy, E., et al. 2003, AJ, 125, 684

Shetrone, M. D., Côté, P., \& Sargent, W. L. W. 2001, ApJ, 548, 592

Skúladóttir, Á, Tolstoy, E., Salvadori, S., Hill, V., \& Pettini, M. 2017, A\&A, 606, A71

Sneden, C., Bean, J., Ivans, I., Lucatello, S., \& Sobeck, J. 2012, MOOG: LTE Line Analysis and Spectrum Synthesis, Astrophysics Source Code Library, ascl:1202.009

Sneden, C. A. 1973, PhD thesis, Univ. Texas Austin.

Tang, S., Bildsten, L., Wolf, W. M., et al. 2014, ApJ, 786, 61

Timmes, F. X., Brown, E. F., \& Truran, J. W. 2003, ApJL, 590, L83

Timmes, F. X., Woosley, S. E., \& Taam, R. E. 1994, ApJ, 420, 348

Townsley, D. M., Miles, B. J., Shen, K. J., \& Kasen, D. 2019, ApJ, 878, L38

Tucker, M. A., Shappee, B. J., Vallely, P. J., et al. 2019, MNRAS, submitted (arXiv:1903.05115)

Ural, U., Cescutti, G., Koch, A., et al. 2015, MNRAS, 449, 761

van Kerkwijk, M. H., Chang, P., \& Justham, S. 2010, ApJL, 722, L157

Venn, K. A., Irwin, M., Shetrone, M. D., et al. 2004, AJ, 128, 1177

Venn, K. A., Shetrone, M. D., Irwin, M. J., et al. 2012, ApJ, 751, 102

Vennes, S., Nemeth, P., Kawka, A., et al. 2017, Sci, 357, 680

Webbink, R. F. 1984, ApJ, 277, 355

Weisz, D. R., Dolphin, A. E., Skillman, E. D., et al. 2014, ApJ, 789, 147

Wheeler, J. C., Sneden, C., \& Truran, J. W., Jr. 1989, ARA\&A, 27, 279

Whelan, J., \& Iben, I. J. 1973, ApJ, 186, 1007

White, C. J., Kasliwal, M. M., Nugent, P. E., et al. 2015, ApJ, 799, 52

Woosley, S. E., Taam, R. E., \& Weaver, T. A. 1986, ApJ, 301, 601

Woosley, S. E., \& Weaver, T. A. 1995, ApJS, 101, 181

Yamaguchi, H., Badenes, C., Foster, A. R., et al. 2015, ApJL, 801, L31

Yoon, S. C., \& Langer, N. 2003, A\&A, 412, L53

Yungelson, L. R. 2010, AstL, 36, 780

Yungelson, L. R., \& Livio, M. 2000, ApJ, 528, 108 